\title{
Acceleration Properties of \\ Planar Manipulators \\ from a Dynamic Standpoint
}

Yong-yil Kim and Subhas Desa

CMU-RI-TR-88-16

The Robotics Institute

Carnegie Mellon University

Pittsburgh, Pennsylvania 15213

September 1988

CC 1988 Carnegie Mellon University 



\section{Table of Contents}

1 Introduction

2 Definition of the Problem

2.1 Definition of the manipulator variables

2.2 Manipulator acceleration

2.3 Definition of the problem

3 Decomposition of the manipulator jacobian

4 Linear mapping

5 Quadratic mapping

5.1 Description of $S$.

5.1.1 The quadratic map and the description of $S_{y}$

5.1.2 The linear mapping (96) and the determination of $S_{\dot{q}}$

5.2 Properties of $S_{\dot{q}}$

5.2.1 Furthest vertex of $S_{\dot{q}}$

5.2.2 Supremum of $S_{\dot{q}}$

5.2.3 Infimum of $S_{\dot{q}}$

6 The state acceleration set, $S_{u}$

6.1 Determination of $S_{u}$

7 Local acceleration sets

7.1 Determination of $\left(\mathrm{S}_{\mathrm{L}}\right)_{1}$

7.2 Supremum of $\left(\mathrm{S}_{\mathrm{L}}\right)_{1}$

7.3 Determination of $\left(S_{L}\right)_{2}$

7.4 Infimum of $\left(S_{L}\right)_{2}$ 



\section{List of Figures}

Figure 1: A two degree-of-freedom manipulator

Figure 2: Description of the velocity vector.

Figure 3: Linear Mapping

Figure 4: an available joint velocity set

Figure 5: Image set of $\mathrm{O}_{1} \mathrm{H}_{1} \mathrm{E}_{1}$

Figure 6: Image set of $O_{1} E_{1} G_{1}$

Figure 7: Image set of $O_{1} E_{1} G_{1}$

Figure 8: Image set of $\mathrm{O}_{1} \mathrm{~F}_{1} \mathrm{H}_{2}$

Figure 9: Image set $\mathrm{S}_{\mathrm{u}}$

Figure 10: Approximation of Image set

Figure 11: Image set $S_{\dot{q}} \quad 22$

Figure 12: A state acceleration set $\quad 25$

Figure 13: the relative location of a parallelogram to the origin 26

Figure 14: Determination of $\left(S_{U}\right)_{1}$

Figure 15: A local supremum 29

Figure 16: $\left(S_{L}\right)_{2}$ and a local infimum 30

Figure 17: The acceleration set for a dynamic state 0

Figure 18: The local acceleration set 33 



\begin{abstract}
An important issue in designing manipulators for dynamic performance is the determination of the acceleration properties of (some reference-point on) the end-effector of the manipulator. Given the dynamical equations of the planar two degree-of-freedom manipulator and a set of constraints on the actuator torques and on the rates-ofchanges of the joint variables, we systematically develop (a) the properties of the linear mapping between the actuator torques and the acceleration of (some reference-point on) the end-effector and (b) the properties of the (non-linear) quadratic mapping between the rates-of-changes of the joint variables and the acceleration of the end-effector. We then show how these mappings can be combined to obtain useful acceleration sets - for example the acceleration set corresponding to any point in the workspace of the manipulator - as well as the properties of these sets.
\end{abstract}





\section{Introduction}

An important issue in designing manipulators for dynamic performance is the determination of the acceleration properties of (some reference-point on) the end-effector of the manipulator. Given the dynamical equations of the planar two degree-of-freedom manipulator and a set of constraints on the actuator torques and on the rates-of-changes of the joint variables, we systematically develop (a) the properties of the linear mapping between the actuator torques and the acceleration of (some reference-point on) the end-effector and (b) the properties of the (non-linear) quadratic mapping between the rates-of-changes of the joint variables and the acceleration of the end-effector. We then show how these mappings can be combined to obtain useful acceleration sets - for example the acceleration set corresponding to any point in the workspace of the manipulator - as well as the properties of these sets.

It is useful to briefly mention the work done by others on related problems. Khatib [1,2] sets up an optimization problem to improve the acceleration of the endeffector; however the non-linear terms in the dynamical equations are accounted for in somewhat ad-hoc fashion (by taking certain "high" and "low" values of these terms). Graettinger and Krogh [3] use semi-infinite programming to obtain the "acceleration-radius" (or "isotropic acceleration") of a manipulator. In contrast to our approach, both the above-mentioned approaches do not yield the acceleration sets of the manipulators (defined in section 2) or the properties of these sets or show how the properties of the sets are related to the geometric and dynamical parameters of the manipulator.

The report is organized as follows: The problem and the variables of interest (in the problem) are defined in section 2 . In the next section, we describe certain decompositions of the manipulator Jacobians which are useful in deriving certain properties of the linear and (non-linear) quadratic mappings. The properties of the linear mapping and the quadratic mapping, respectively, are derived in section 4 and 5 . In section 6, we show how these maps can be combined to obtained the acceleration set corresponding to any point in the state space; we then determine the properties of this set. Similarly, section 7 is devoted to determining the acceleration set (and its properties) corresponding to any configuration (or "position") in the workspace of the manipulator. In the final section, using the example of the planar manipulator in our laboratory, we address the computation of the acceleration sets and their properties. 


\section{Definition of the Problem}

In this section, using a simple two degree-of-freedom manipulator, we define the problem to understand the dynamic performance of manipulators.

First, we define manipulator variables in subsection 2.1. In the following subsection, we express the manipulator acceleration in terms of the variables. In subsection 2.3 , we define the problem to characterize the manipulator performance with end-effector acceleration.

\subsection{Definition of the manipulator variables}

Consider the serial two degree-of-freedom manipulator with two revolute joints shown in Figure 1. The manipulator is assumed to be rigid with negligible joint friction.

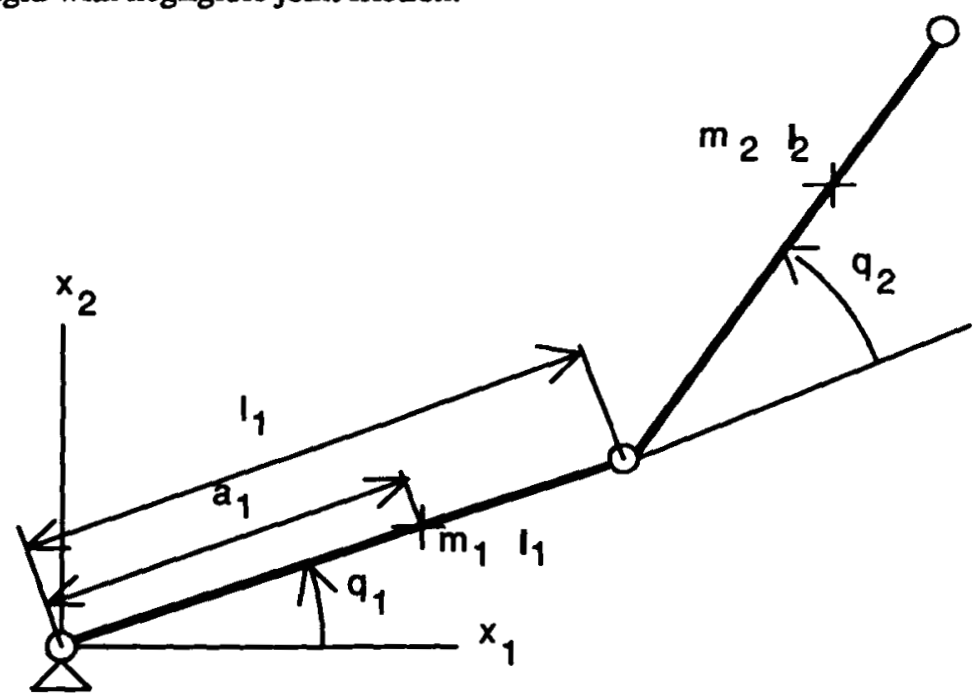

Figure 1: A two degree-of-freedom manipulator

Let $l_{1}$ denote the length of link $1, a_{1}$ the distance from joint axis 1 to the center of mass of link $1, m_{1}$ the mass of link 1 and $I_{1}$ the principal moment of inertia of link 1 with respect to its center of mass about an axis perpendicular to the plane of the motion. Similarly, let $l_{2}, a_{2}, \mathrm{~m}_{2}$ and $\mathrm{I}_{2}$ denote the corresponding properties of link 2 (See Figure 1). We call these quantities design variables.

$\tau_{1}$ and $\tau_{2}$ denote the joint torques, respectively, at joints 1 and 2 and

$$
\tau=\left[\begin{array}{ll}
\tau_{1} & \tau_{2}
\end{array}\right]^{T}
$$

denotes the vector of joint torque vectors. Let

$$
\left|\tau_{i}\right| \leq \tau_{i o}, i=1,2
$$

denote the constraints on the actuator torques at joints 1 and 2 . We define

to be the set of allowable torques.

$$
T=\left\{\tau|| \tau_{i} \mid \leq \tau_{i 0}, i=1,2\right\}
$$

Let $\left(x_{1}, x_{2}\right)$ denote the coordinates, in a reference frame fixed to the base, of a reference point $P$ on link 2 (See 
Figure 1) and define

$$
\mathbf{x}=\left[\begin{array}{ll}
x_{1} & x_{2}
\end{array}\right]^{T}
$$

to be the vector of task coordinates in task space. Let $q_{1}$ and $q_{2}$ denote the generalized coordinates of the manipulator (See Figure 1), $q_{1}$ being the joint variable at joint 1 and $q_{2}$ the joint variable at joint 2 . Define

$$
\mathbf{q}=\left[\begin{array}{ll}
q_{1} & q_{2}
\end{array}\right]^{T}
$$

to be the vector of joint variables in joint space. If

$$
q_{i L} \leq q_{i} \leq q_{i U}, i=1,2
$$

denotes the constraint on joint variable $i$, then we can define the workspace $w$ of a manipulator as

$$
\mathrm{W}=\left\{\mathbf{q} \mid q_{i L} \leq q_{i} \leq q_{i U}, i=1,2\right\}
$$

Let $\dot{q}_{1}$ and $\dot{q}_{2}$ denote the joint velocities (the rates of change of the joint variables) $q_{1}$ and $q_{2}$, respectively. Define

$$
\dot{\mathbf{q}}=\left[\begin{array}{ll}
\dot{q}_{1} & \dot{q}_{2}
\end{array}\right]^{T}
$$

to be the vector of joint velocities. If

$$
\left|\dot{q}_{i}\right| \leq \dot{q}_{i o}, i=1,2
$$

denotes the constraints on the rates of changes of the joint variables, then we can define

$$
\mathbf{F}=\left\{\dot{\mathbf{q}}|| \dot{q}_{i} \mid \leq \dot{q}_{i o}, i=1,2\right\}
$$

to be the set of all possible joint velocity vectors.

Define the state vector

$$
\mathbf{u}=(\mathbf{q} \quad \dot{\mathbf{q}})=\left[\begin{array}{llll}
q_{1} & q_{2} & \dot{q}_{1} & \dot{q}_{2}
\end{array}\right]^{T}
$$

to represent the dynamic state of a manipulator in the state-space.

The acceleration space or acceleration plane, $A$, of the manipulator is the set of all possible accelerations, $\ddot{\mathbf{x}}=\left[\begin{array}{ll}\ddot{x}_{1} & \ddot{x}_{2}\end{array}\right]^{T}$, where $\ddot{x}_{1}$ and $\ddot{x}_{2}$ are real numbers.

More formally we can define

$$
A=\left\{\ddot{x} \mid \ddot{x} \in R^{2}\right\}
$$

where $R^{2}$ is the real Euclidean plane.

\subsection{Manipulator acceleration}

In this subsection, we derive an expression for the acceleration [4],

$$
\ddot{\mathbf{x}}=\left[\begin{array}{ll}
\ddot{x}_{1} & \ddot{x_{2}}
\end{array}\right]^{T},
$$

of the reference-point $P$ on the end-effector, since this quantity plays an important role in our analysis.

The relationship between the velocity, $\dot{x}$, of point $P$, and the "joint velocity" vector $\dot{q}$ is well known:

$$
\dot{\mathbf{x}}=\mathbf{J} \dot{\mathbf{q}}
$$

where $\mathbf{J}$ is a $(2 \times 2)$ matrix called the manipulator Jacobian. The detailed expression of Jacobian matrix is shown in Appendix. 
The dynamic behavior of the two degree-of-freedom manipulator is described by the following equations:

$$
\begin{aligned}
& d_{11}\left(q_{2}\right) \ddot{q}_{1}+d_{12}\left(q_{2}\right) \ddot{q}_{2}-v\left(q_{2}\right)\left(\dot{q}_{1}^{2}+2 \dot{q}_{1} \dot{q}_{2}\right)+p_{1}(q)=\tau_{1} \\
& d_{21}\left(q_{2}\right) \ddot{q}_{1}+d_{22} \ddot{q}_{2}-v\left(q_{2}\right)\left(\dot{q}_{1}^{2}\right)+p_{2}(q)=\tau_{2}
\end{aligned}
$$

where the coefficients $d_{i j}\left(q_{2}\right),(i, j=1,2), v\left(q_{2}\right), p_{i}(q),(i=1,2)$ are given in Appendix; note that coefficients $d_{i j}(i, j$ $=1,2), v\left(q_{2}\right)$ are functions of only joint variable $q_{2}$.

Defining

$$
\begin{aligned}
& \mathbf{D}\left(\mathrm{q}_{2}\right):=\left[\begin{array}{ll}
d_{11}\left(q_{2}\right) & d_{12}\left(q_{2}\right) \\
d_{21}\left(q_{2}\right) & d_{22}
\end{array}\right], \\
& \mathbf{v}\left(\mathrm{q}_{2}\right):=\left[\begin{array}{lll}
0 & -v\left(q_{2}\right) & \vdots \\
v\left(q_{2}\right) & 0
\end{array}\right] \text {, } \\
& \mathrm{p}(\mathbf{q})=\left[p_{1}(\mathbf{q}) p_{2}(q)\right]^{T} \\
& \ddot{\mathbf{q}}=\left[\begin{array}{ll}
\ddot{q}_{1} & \ddot{q}_{2}
\end{array}\right]^{T}
\end{aligned}
$$

and

$$
\dot{q}^{2}=\left[\dot{q}_{1}^{2}\left(\dot{q}_{1}+\dot{q}_{2}\right)^{2}-\dot{q}_{1}^{2}\right]^{T}
$$

the dynamic equations (15) and (16) become,

$$
\mathbf{D}\left(q_{2}\right) \ddot{\mathbf{q}}+\mathbf{V}\left(q_{2}\right)\{\dot{\mathbf{q}}\}^{2}+\mathbf{p}(\mathbf{q})=\tau
$$

The matrix $D\left(q_{2}\right)$ is the mass matrix of the manipulator and the vector $p(q)$ denotes the gravitational terms influencing the dynamic behavior.

A crucial step in the acceleration analysis is the definition of the skew-symmetric matrix $V\left(q_{2}\right)$ and the vector $\{\dot{q}\}^{2}$, which allows all the non-linear terms (often called Coriolis and centrifugal) to be written as the product of $\mathbf{V}\left(\mathrm{q}_{2}\right)$ and $\{\dot{q}\}^{2}$. The notation (\}$^{2}$ is used to draw attention to the fact that the elements of the vector $\{\dot{q}\}^{2}$ are quadratic in the rates-of- changes $\dot{q}_{1}$ and $\dot{q}_{2}$, respectively, of the joint variables $q_{1}$ and $q_{2}$. Note that $\{\dot{q}\}^{2}$ is not equal to $\dot{\mathbf{q}}^{2}=\dot{\mathrm{q}}_{1}{ }^{2}+\dot{\mathrm{q}}_{2}{ }^{2}$.

To obtain the expression for the acceleration $\ddot{x}$ of the point $P$, we differentiate (14) to obtain

$$
\ddot{\mathbf{x}}=\mathbf{J} \ddot{\mathbf{q}}+\dot{j} \dot{q}
$$

In Appendix, we show that the second term in (23), $\mathbf{j} \dot{\mathbf{q}}$, can be written in the form

$$
\dot{\mathbf{J}} \dot{\mathbf{q}}=-\mathbf{E}(\mathbf{q})\{\dot{\mathbf{q}}\}^{2}
$$

Combining (23) and (24) we obtain

$$
\ddot{\mathbf{x}}=\mathbf{J} \ddot{\mathbf{q}}-\mathbf{E}(\mathbf{q})\{\dot{\mathbf{q}}\}^{2} \text {. }
$$

Defining the quantities,

$$
\mathbf{A}(\mathbf{q})=\mathbf{J}(\mathbf{q}) \mathbf{D}^{-1}\left(q_{2}\right),
$$




$$
\begin{aligned}
& \mathbf{B}(\mathbf{q})=-\mathbf{A}(\mathbf{q}) \mathbf{V}(\mathbf{q})-\mathbf{E}(\mathbf{q}), \\
& \mathbf{c}(\mathbf{q})=-\mathbf{A}(\mathbf{q}) \mathbf{p}(\mathbf{q}),
\end{aligned}
$$

it is easy to verify that the expression for the acceleration $\ddot{x}$ of the point $P$, obtained by combining equation (22) with equations (25) through (28), is given by

$$
\ddot{\mathbf{x}}=\mathbf{A}(\mathbf{q}) \tau+\mathbf{B}\{\dot{q}\}^{2}+\mathbf{c}(\mathbf{q}) .
$$

Note that $A(q), B(q)$ and $c(q)$ are position dependent, the expressions for the coefficients of which are given in Appendix.

If the manipulator operates in a (horizontal) plane perpendicular to gravity, then $\mathbf{c}(\mathbf{q})=\mathbf{0}$ and (29) becomes

$$
\ddot{\mathbf{x}}=\mathbf{A}(\mathbf{q}) \boldsymbol{\tau}+\mathbf{B}[\dot{\mathbf{q}}]^{2} \text {. }
$$

In this paper, we will study manipulators, moving in horizontal planes, whose acceleration properties are described by equation (30).

Defining

$$
\alpha_{\tau}=\left[\begin{array}{ll}
\alpha_{1 \tau} & \alpha_{2 \tau}
\end{array}\right]^{T}=\mathbf{A}(\mathbf{q}) \tau
$$

and

equation (30) can be written as

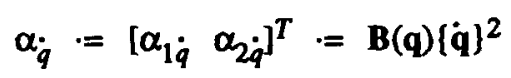

$$
\ddot{\mathbf{x}}=\alpha_{\mathrm{r}}+\alpha_{\dot{q}}
$$

It is convenient to think of $\alpha_{\tau}$ as the contribution of the torques to the acceleration of the reference point $P$ and $\alpha_{\dot{q}}$ as the contribution of the joint-rates to the acceleration of $P$, the sum of these two quantities giving us the acceleration of $P$ as expressed by equation (33).

Equation (31) can be viewed as a linear, position-dependent, mapping between the torque vector $\tau$ and its contribution $\alpha_{\tau}$ to the acceleration of P. Similarly equation (32) can be viewed as a quadratic, position-dependent, mapping between the joint rate vector $\dot{q}$ and its contribution $\alpha_{\dot{q}}$ to the acceleration of $P$.

\subsection{Definition of the problem}

The acceleration $\ddot{x}$ of the reference point $P$ of a manipulator, specified by its design variables, constraints on the torques as given by (2) or (3), constraints on the joint variables as given by (6) or (7) and constraints on the joint velocities as given by (9) or (10), will be a subset of the acceleration plane A of equation (12). In other words, the acceleration set for a combination of the above constraints can represent the dynamic performance of manipulators. To characterize the manipulator dynamic performance, we generate four acceleration sets as follows:

First, we consider the manipulator acceleration set when the joint velocity is zero. Physically, the set represents the manipulator dynamics when a manipulator starts to move. For the given set $T$ of allowable actuator torques described by (3), we define the set of all allowable $\alpha_{\tau}$ as

$$
s_{\tau}=\left\{\alpha_{\tau} \mid(\exists \tau \in T)\left(\alpha_{\tau}=A \tau\right)\right\}
$$


Next, when the actuator torque vanishes during the operation of a manipulator, the subsequent motion of a manipulator is also critical in manipulator dynamics. For the given (constraint) set $\mathrm{F}$ of allowable rates-of-change, described by (10), we define the set $S_{\dot{q}}$ of all allowable $\alpha_{\dot{q}}$ as

$$
s_{\dot{q}}=\left\{\alpha_{\dot{q}} \mid(\exists \dot{q} \in F)\left(\alpha_{\dot{q}}=B[\dot{q}\}^{2}\right)\right\}
$$

Finally, when a manipulator is in motion, we consider two acceleration sets in the following. The acceleration of the reference point $P$ corresponding to a specified state-vector $u=\left[q_{1}, q_{2}, \dot{q}_{1}, \dot{q}_{2}\right]^{T}$ in the state-space will be denoted by $\alpha_{u}$. From equation (30), we write

$$
\alpha_{u}=\mathbf{A}(\mathbf{q}) r+\mathbf{B}(\mathbf{q})[\dot{q}\}^{2}
$$

If we define a constant vector $k$,

$$
\mathbf{k}(\mathbf{u})=\mathbf{k}(\mathbf{q}, \dot{\mathbf{q}})=\left[k_{1}, k_{2}\right]^{T}=\alpha_{\dot{\mathbf{q}}}(\mathbf{q}, \dot{\mathbf{q}})=\mathbf{B}(\mathbf{q})[\dot{\mathbf{q}}]^{2},
$$

then (36) can be written as

$$
\alpha_{\mathbf{u}}=\mathbf{A}(\mathbf{q}) \tau+\mathbf{k}(\mathbf{u})
$$

We now define the acceleration set, $S_{u}$, at a specified point $u$ in the state space as follows: For a given set $T$ of allowable actuator torques described by (3), the acceleration set $S_{u}$ at a point $\mathbf{u}=[\mathbf{q}, \dot{\mathbf{q}}]^{T}$ in the state - space is given by

$$
S_{u}(\mathbf{q}, \dot{\mathbf{q}})=\left\{\alpha_{u} \mid(u=(\mathbf{q}, \dot{\mathbf{q}})),(\exists \tau \in T)\left(\tau_{u}=\mathbf{A}(\mathbf{q}) \tau+k(u)\right)\right\}
$$

Thus $S_{u}$ is the image of the set $T$ under the mapping (38).

Finally, at a given position $q=\left[q_{1}, q_{2}\right]^{T}$ in the workspace of the manipulator, we can define two sets

$$
\begin{aligned}
& \left(s_{L}\right)_{1}=\cup_{\dot{q} \in F} s_{u}(q, \dot{q}) \\
& \left(s_{L}\right)_{2}=\cap_{\dot{q} \in F} s_{u}(q, \dot{q})
\end{aligned}
$$

The supremum of $\left(S_{L}\right)_{1}$ will be give us the magnitude of the maximum acceleration (in some direction) of the reference point $P$ at a given position $\left(q_{1}, q_{2}\right)$ of the manipulator.

The infimum of $\left(S_{L_{2}}\right.$ will give us the magnitude of the maximum acceleration of the reference point $P$ available in all direction at a given position of the manipulator. The infimum of $\left(\mathrm{S}_{L}\right)_{2}$ is called the isotropic acceleration in Khatib [1] and the local acceleration radius in Kim [5].

Based on the above definitions, the manipulator problem can be written as follows;

1. To characterize four acceleration sets, $s_{\tau}, s_{q}, s_{u}, s_{L}$, with their shape and supremum and infimum.

2. To examine the behavior of four acceleration sets $s_{\tau}, s_{\dot{q}}, s_{u}, s_{L}$.

In Section 4 and 5, respectively, we study the properties of the linear mapping described by equation (31) and the properties of the non-linear mapping described by equation (32). The acceleration properties of $P$, obtained by combining these two maps, is discussed in Section 6. In the next section we present a decomposition of the Jacobian which is helpful in the study of the aforementioned linear and non-linear maps. 


\section{Decomposition of the manipulator jacobian}

In this section, we derive some little known decompositions of the manipulator Jacobians. These decompositions facilitate the derivation of the properties of the acceleration properties in Sections 4 and 5. Let $v$ denote the velocity of point $P$ in a reference frame fixed to the base $N,\left(n_{1} n_{2}\right)$ be a set of dextral orthogonal unit vectors fixed in $N,\left(a_{1} a_{2}\right)$ a set of dextral orthogonal unit vectors fixed in $A$, and $\left(\begin{array}{ll}b_{1} & b_{2}\end{array}\right)$ a set of dextral orthogonal vectors fixed in $B$ (see Figure 2).

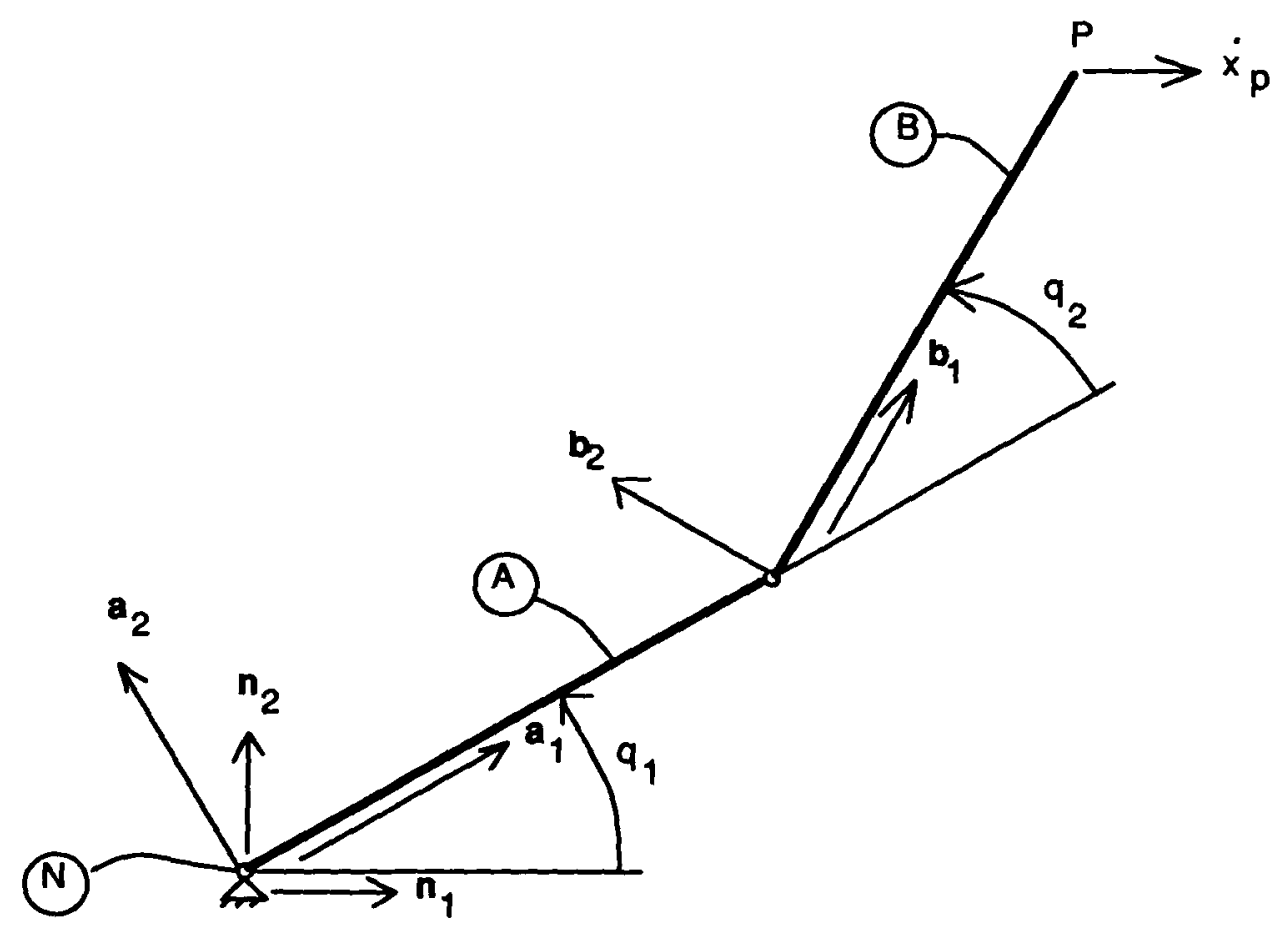

Figure 2: Description of the velocity vector.

We pick $n_{1}$ so that it points in the direction of the positive $x_{1}$ axis and pick $n_{2}$ so that it points in the direction of the positive $x_{2}$ axis (Figure 2).

Defining,

$$
\left.\mathbf{R}\left(\mathrm{q}_{i}\right):=\begin{array}{lll}
\cos q_{i} & -\sin q_{i} \\
& & \\
\sin q_{i} & \cos q_{i}
\end{array}\right], \quad(\mathrm{i}=1,2)
$$

and referring to Figure 2, we obtain,

$$
\begin{aligned}
& {\left[\begin{array}{ll}
\mathbf{a}_{1} & \mathbf{a}_{2}
\end{array}\right]^{T}=\mathbf{R}\left(q_{2}\right)\left[\begin{array}{ll}
\mathbf{b}_{1} & \mathbf{b}_{2}
\end{array}\right]^{T}} \\
& {\left[\begin{array}{ll}
\mathbf{n}_{1} & \mathbf{n}_{2}
\end{array}\right]^{T}=\mathbf{R}\left(q_{1}\right)\left[\begin{array}{ll}
\mathbf{a}_{1} & \mathbf{a}_{2}
\end{array}\right]^{T} .}
\end{aligned}
$$

The velocity of the reference point, $v$, in the refenence frame $\mathrm{N}$ is given by

$$
\mathbf{v}=l_{1} \dot{q}_{1} \mathbf{a}_{2}+l_{2}\left(\dot{q}_{1}+\dot{q}_{2}\right) \mathbf{b}_{2}
$$

From equation (45), we obtain 


$$
\begin{aligned}
& \mathbf{v} \bullet \mathbf{b}_{1}=l_{1} \dot{q}_{1} \mathbf{a}_{2} \bullet \mathbf{b}_{1} \\
& \mathbf{v} \bullet \mathbf{b}_{2}=l_{1} \dot{q}_{1} \mathbf{a}_{2} \bullet \mathbf{b}_{2}+l_{2}\left(\dot{q}_{1}+\dot{q}_{2}\right) .
\end{aligned}
$$

Note that $\mathbf{v} \bullet b_{1}$ and $v \bullet b_{2}$ are simply the $b_{1}$ and $b_{2}$ measure numbers of the velocity of $P$ in $N$. Using equations (42), equations (46) and (47) can be rewritten in the matrix form,

Defining,

$$
\begin{aligned}
& \left\lceil\operatorname{veb}_{1}\right\rceil \quad\left\lceil l_{1} \sin q_{2} \quad 0 \quad\right\rceil\left\lceil\dot{q}_{1}\right\rceil \\
& \left\lfloor v \bullet b_{2}\right\rfloor \quad\left\lfloor l_{1} \cos q_{2}+l_{2} \quad l_{2}\right\rfloor\left\lfloor\dot{q}_{2}\right\rfloor .
\end{aligned}
$$

$$
\left.\mathbf{M}\left(\mathrm{q}_{2}\right):=\begin{array}{lll}
l_{1} \sin q_{2} & 0 & \rceil \\
& l_{1} \cos q_{2}+l_{2} & l_{2}
\end{array}\right]
$$

and using (5), equation (48) becomes

$$
\left[\begin{array}{ll}
\mathbf{v} \bullet b_{1} & \mathbf{v} \bullet b_{2}
\end{array}\right]^{T}=\mathbf{M}\left(q_{2}\right) \dot{q} \text {. }
$$

Referring again to the geometry of Figure 2, we can write,

$$
\begin{aligned}
& {\left[\begin{array}{ll}
\mathbf{v} \bullet a_{1} & v \bullet a_{2}
\end{array}\right]^{T}=\mathbf{R}\left(q_{2}\right)\left[\begin{array}{lll}
v \bullet b_{1} & v \bullet b_{2}
\end{array}\right]^{T}} \\
& {\left[\begin{array}{lll}
\mathbf{v} \bullet \mathrm{n}_{1} & \mathbf{v} \bullet \mathrm{n}_{2}
\end{array}\right]^{T}=\mathbf{R}\left(q_{1}\right)\left[\begin{array}{ll}
\mathbf{v} \bullet \mathrm{a}_{1} & \mathbf{v} \bullet \mathrm{a}_{2}
\end{array}\right]^{T}}
\end{aligned}
$$

Equation (51) simply relates the $b_{1}$ and $b_{2}$ measure numbers of $v$ to the $a_{1}$ and $a_{2}$ measure numbers of $v$; similarly (52) relates the $a_{1}$ and $a_{2}$ measure numbers of $v$ to the $n_{1}$ and $n_{2}$ measure numbers of $v$.

Combining equations (50) through (52) we obtain

$$
\left[\begin{array}{ll}
\mathbf{v} \bullet n_{1} & \mathbf{v} \bullet n_{2}
\end{array}\right]=\mathbf{R}\left(q_{1}\right) \mathbf{R}\left(q_{2}\right) \mathbf{M}\left(q_{2}\right) \dot{q}
$$

If $x$ is the position vector of $P$ with respect to the fixed point pivot $O$, then from equation (4) and the choice of the directions of $\mathbf{n}_{1}$ and $\mathbf{n}_{2}$ (see Figure 2), we can write

and,

$$
\mathbf{x}=x_{1} \mathbf{n}_{1}+x_{2} n_{2}
$$

$$
\mathbf{v}=\dot{\mathbf{x}}=\dot{x}_{1} \mathbf{n}_{1}+\dot{x}_{2} \mathbf{n}_{2}
$$

From (55), the $n_{1}$ and $n_{2}$ measure numbers of $v$ are given by

$$
\left[\begin{array}{ll}
v \bullet n_{1} & v \bullet n_{2}
\end{array}\right]^{T}=\left[\begin{array}{ll}
\dot{x}_{1} & \dot{x}_{2}
\end{array}\right]^{T}
$$

Combining (53) and (56) we obtain,

$$
\dot{\mathbf{x}}=\left[\begin{array}{ll}
\dot{x}_{1} & \dot{x}_{2}
\end{array}\right]^{T}=\mathbf{R}\left(q_{1}\right) \mathbf{R}\left(q_{2}\right) \mathbf{M}\left(q_{2}\right) \dot{\mathbf{q}}
$$

Comparing equations (57) and (14), we obtain

$$
\mathbf{J}(q)=\mathbf{R}\left(q_{1}\right) \mathbf{R}\left(q_{2}\right) \quad \mathbf{M}\left(q_{2}\right)
$$

Note that we have decomposed the Jacobian matrix, $J(q)$, into the product of three matrices which depend either on $q_{1}$ or $q_{2}$ but not both $q_{1}$ and $q_{2}$. $R\left(q_{1}\right)$ and $\mathbf{R}\left(q_{2}\right)$ are simple orthogonal matrices and $N\left(q_{2}\right)$ represents the kinematic 
coupling between the two links. In a similar fashion, we can show that the matrix $\mathbf{E}(\mathbf{q})$ in equation (24) can be written as

$$
\mathbf{E}(\mathbf{q})=\mathbf{R}\left(q_{1}\right) \mathbf{R}\left(q_{2}\right) \mathbf{N}\left(q_{2}\right)
$$

where

$$
\mathbf{N}\left(q_{2}\right):=\left[\begin{array}{ccc}
l_{1} \cos q_{2}+l_{2} & l_{2} & \rceil \\
-l_{1} \sin q_{2} & 0
\end{array}\right]
$$

Equations (58) and (59) are very useful in deriving the properties of the acceleration maps in section 4 and 5. 


\section{Linear mapping}

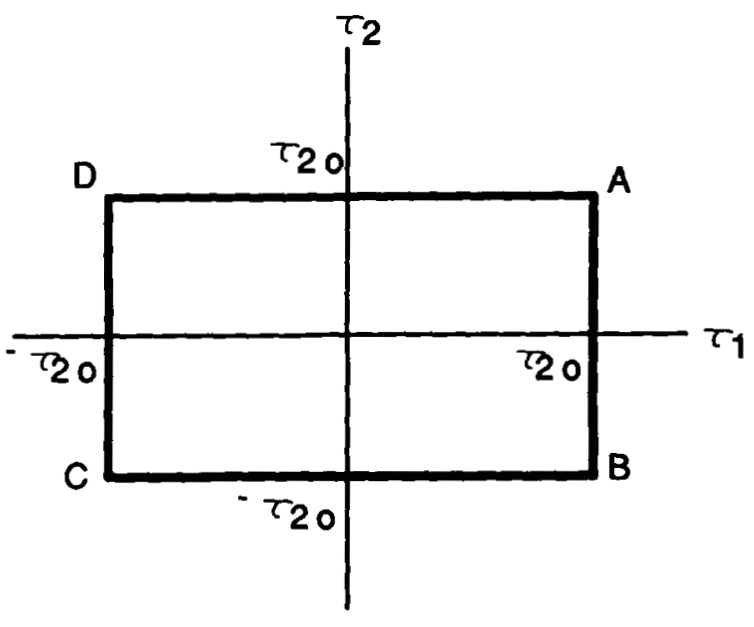

(a)

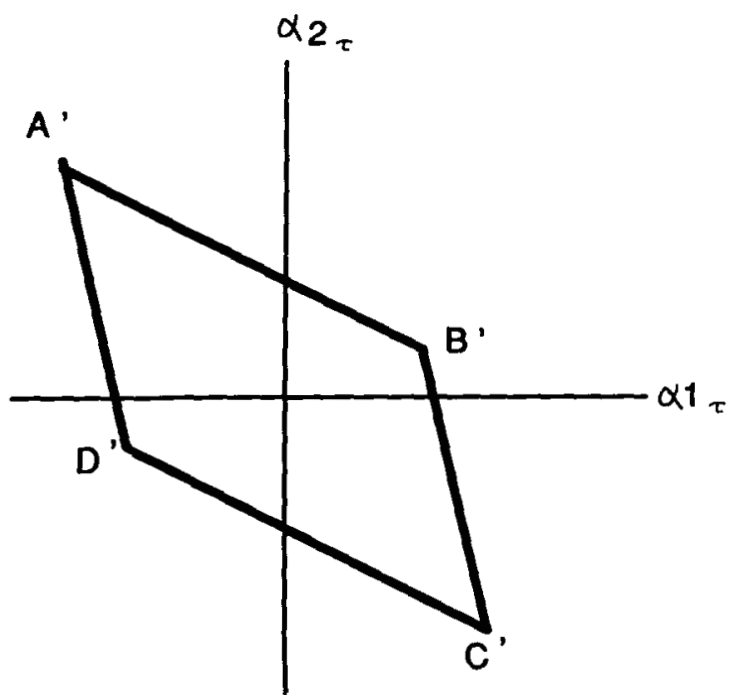

(b)

Figure 3: Linear Mapping

For the given set $\mathrm{T}$ of allowable actuator torques described by (3) and represented by ( the interior and the boundary of ) the rectangle $A B C D$ shown in Figure 3-a, we defined the set of all allowable $\alpha_{\tau}$ in section 2 as

$$
S_{\tau}=\left\{\alpha_{\tau} \mid(\exists \tau \in T)\left(\alpha_{\tau}=A \tau\right)\right\}
$$

In this section, we simply state that $S_{\tau}$ is the parallelogram $A^{\prime} B^{\prime} C^{\prime} D^{\prime}$ shown in Figure 3-b and we will derive three properties of the set $S_{\tau}$. The first property determines the "size" of $S_{\tau}$, as characterized by its infimum, supremum, and its area. The second property expresses the $q_{1}$ invariance of $s_{\tau}$ The third property expresses the $q_{2}$ dependency of $\mathrm{s}_{\tau}$.

The decomposition, expressed by equation (58), of the manipulator Jacobian is extremely useful in the derivation of the properties of the set $S_{\tau}$. Combining equation (58) and (26) we obtain

$$
\mathbf{A}(\mathbf{q})=\mathbf{R}\left(q_{1}\right) \mathbf{R}\left(q_{2}\right) \quad \mathbf{M}\left(q_{2}\right) \mathbf{D}^{-1}\left(q_{2}\right) .
$$

Defining a matrix $\mathbf{P}\left(\mathbf{q}_{2}\right)$ as

$$
\mathbf{P}\left(q_{2}\right)=\mathbf{R}\left(q_{2}\right) \mathbf{M}\left(q_{2}\right) \mathbf{D}^{-1}\left(q_{2}\right),
$$

we can rewrite (61) as

$$
\mathbf{A}(\mathbf{q})=\mathbf{R}\left(q_{1}\right) \mathbf{P}\left(q_{2}\right)
$$

Note that we have decomposed $A(q)$ into an orthogonal matrix $R\left(q_{1}\right)$ depending only on $q_{1}$ and a matrix $P\left(q_{2}\right)$ depending only on $q_{2}$. This decomposition facilitates the derivation of the properties below. The (i,j) element of $A$ and $\mathbf{P}$ will be denoted, respectively, by $a_{i j}$, and $p_{i j}$.

Let $A^{\prime}, B^{\prime}, C^{\prime}, D^{\prime}$ denote, respectively, the points in the $\alpha_{\tau}$ - plane into which the points $A\left(\tau_{1 o^{\prime}}, \tau_{20}\right), B\left(\tau_{1 o^{-}}\right.$ $\left.\tau_{20}\right), C\left(-\tau_{10},-\tau_{20}\right)$, and $D\left(-\tau_{10}, \tau_{20}\right)$ map. Then it is easy to verify that the coordinates of $A^{\prime}, B^{\prime}, C^{\prime}, D^{\prime}$ in the $\alpha_{\tau}$ - 
plane are given by

$$
\begin{aligned}
& A^{\prime}\left(a_{11} \tau_{10}+a_{12} \tau_{20} a_{21} \tau_{10}+a_{22} \tau_{20}\right) \\
& B^{\prime}\left(a_{11} \tau_{10}-a_{12} \tau_{20} a_{21} \tau_{10}-a_{22} \tau_{20}\right) \\
& C^{\prime}\left(-a_{11} \tau_{10}-a_{12} \tau_{20}-a_{21} \tau_{10}-a_{22} \tau_{20}\right) \\
& D^{\prime}\left(-a_{11} \tau_{10}+a_{12} \tau_{20}-a_{21} \tau_{10}+a_{22} \tau_{20}\right) \text {. }
\end{aligned}
$$

Since (31) is a linear mapping, $A^{\prime} B^{\prime} C^{\prime} D^{\prime}$ is a parallelogram; the equations of the sides $A^{\prime} B^{\prime}, B^{\prime} C^{\prime}, C^{\prime} D^{\prime}$ and $D^{\prime} A^{\prime}$ are readily obtained as

$$
\begin{aligned}
& A^{\prime} B^{\prime} \frac{1}{a_{12}} a_{1 \tau}-\frac{1}{a_{22}} a_{2 \tau}+\left(-\frac{a_{11}}{a_{12}}+\frac{a_{21}}{a_{22}}\right) \tau_{10}=0,\left(a_{11} \tau_{10}-a_{12} \tau_{20} \leq a_{1 \tau} \leq a_{11} \tau_{10}+a_{12} \tau_{20}\right) \\
& B^{\prime} C^{\prime} \frac{1}{a_{11}} a_{1 \tau}-\frac{1}{a_{21}} a_{2 \tau}+\left(-\frac{a_{12}}{a_{11}}+\frac{a_{22}}{a_{21}}\right) \tau_{20}=0,\left(-a_{11} \tau_{10}+a_{12} \tau_{20} \leq a_{1 \tau} \leq a_{11} \tau_{10}+a_{12} \tau_{20}\right) \\
& C^{\prime} D^{\prime} \frac{1}{a_{12}} a_{1 \tau}-\frac{1}{a_{22}} a_{2 \tau}-\left(-\frac{a_{11}}{a_{12}}+\frac{a_{21}}{a_{22}}\right) \tau_{10}=0,\left(-a_{11} \tau_{10}-a_{12} \tau_{20} \leq a_{1 \tau} \leq-a_{11} \tau_{10}+a_{12} \tau_{20}\right) \\
& D^{\prime} A^{\prime} \quad \frac{1}{a_{11}} a_{1 \tau}-\frac{1}{a_{21}} a_{2 \tau}-\left(-\frac{a_{12}}{a_{11}}+\frac{a_{22}}{a_{21}}\right) \tau_{20}=0,\left(-a_{11} \tau_{10}-a_{12} \tau_{20} \leq a_{1 \tau} \leq a_{11} \tau_{10}-a_{12} \tau_{20}\right) .
\end{aligned}
$$

-Property 1: The set $S_{\tau}$ is a parallelogram whose supremum, infimum and area are given by

$$
\begin{aligned}
& \sup \left(S_{\tau}\right)=\max \left[\sqrt{\left(p_{11}{ }^{2}+p_{21}{ }^{2}\right) \tau_{10}{ }^{2}+\left(p_{12}{ }^{2}+p_{22}{ }^{2}\right) \tau_{20}{ }^{2} \pm 2\left(p_{11} p_{12}+p_{21} p_{22}\right) \tau_{10} \tau_{20}}\right] . \\
& \inf \left(S_{\tau}\right)=\min \left[\frac{\left|\left(p_{11} p_{22}-p_{12} p_{21}\right) \tau_{10}\right|}{\sqrt{p_{12}{ }^{2}+p_{22}{ }^{2}}} \frac{\left|\left(p_{11} p_{22}-p_{12} p_{21}\right) \tau_{20}\right|}{\sqrt{p_{11}{ }^{2}+p_{21}{ }^{2}}}\right] \\
& \operatorname{area}\left(S_{\tau}\right)=\left|\frac{l_{1} l_{2} \sin q_{2}}{\left(I_{1}+m_{1} a_{1}^{2}\right)\left(I_{2}+m_{2} a_{2}{ }^{2}\right)+\left(I_{2}+m_{2} a_{2}{ }^{2} \sin ^{2} q_{2}\right) m_{2} l_{1}^{2}}\right| 4 \tau_{10} \tau_{20}
\end{aligned}
$$

-Proof:

If $\sigma\left(O^{\prime} A^{\prime}\right), \sigma\left(O^{\prime} B^{\prime}\right), \sigma\left(O^{\prime} C^{\prime}\right), \sigma\left(O^{\prime} D^{\prime}\right)$ denote, respectively, the distances of the vertices $A^{\prime}, B^{\prime}, C^{\prime}$, and $D^{\prime}$ from the origin $O^{\prime}$, then the supremum of $S_{\tau}$ is given by

$$
\sup \left(S_{\tau}\right)=\max \left[\sigma\left(O^{\prime} A^{\prime}\right), \sigma\left(O^{\prime} B^{\prime}\right), \sigma\left(O^{\prime} C^{\prime}\right), \sigma\left(O^{\prime} D^{\prime}\right)\right]
$$

Since $A^{\prime}$ and $C^{\prime}$ are equidistant from the origin $O^{\prime}$ and since $B^{\prime}$ and $D^{\prime}$ are also equidistant from the origin, ( observation (1) above ),

$$
\sup \left(s_{\tau}\right)=\max \left[\sigma\left(O^{\prime} A^{\prime}\right), \sigma\left(O^{\prime} B^{\prime}\right)\right]=\frac{1}{2} \max \left[\sigma\left(A^{\prime} C^{\prime}\right), \sigma\left(B^{\prime} D^{\prime}\right)\right]
$$

where $\sigma\left(A^{\prime} C^{\prime}\right)$ and $\sigma\left(B^{\prime} D^{\prime}\right)$ denote the lengths of the diagonals $A^{\prime} C^{\prime}$ and $B^{\prime} D^{\prime}$. Combining equations (64) - (67), (63) and $(42), \sigma\left(A^{\prime} C^{\prime}\right)$ and $\sigma\left(B^{\prime} D^{\prime}\right)$ are given by

Therefore,

$$
\begin{aligned}
& \sigma\left(A^{\prime} C^{\prime}\right)=\sqrt{\left(p_{11}{ }^{2}+p_{21}{ }^{2}\right) \tau_{10}{ }^{2}+\left(p_{12}{ }^{2}+p_{22}{ }^{2}\right) \tau_{20}{ }^{2}+2\left(p_{11} p_{12}+p_{21} p_{22}\right) \tau_{10} \tau_{20}} \\
& \sigma\left(B^{\prime} D^{\prime}\right)=\sqrt{\left(p_{11}{ }^{2}+p_{21}{ }^{2}\right) \tau_{10}{ }^{2}+\left(p_{12}{ }^{2}+p_{22}{ }^{2}\right) \tau_{20}{ }^{2}-2\left(p_{11} p_{12}+p_{21} p_{22}\right) \tau_{10} \tau_{20}}
\end{aligned}
$$

$$
\sup \left(S_{\tau}\right)=\max \left[\sqrt{\left(p_{11}{ }^{2}+p_{21}{ }^{2}\right) \tau_{10}{ }^{2}+\left(p_{12}{ }^{2}+p_{22}{ }^{2}\right) \tau_{20}{ }^{2} \pm 2\left(p_{11} p_{12}+p_{21} p_{22}\right) \tau_{10} \tau_{20}}\right] .
$$

If $\rho\left(O^{\prime} A^{\prime}\right), \rho\left(O^{\prime} B^{\prime}\right), \rho\left(O^{\prime} C^{\prime}\right), \rho\left(O^{\prime} D^{\prime}\right)$ denote, respectively, the distances from $O^{\prime}$ to $A^{\prime} B^{\prime}, B^{\prime} C^{\prime}, C^{\prime} D^{\prime}, D^{\prime} A^{\prime}$, then 
the infimum of $S_{\tau}$ is given by

$$
\inf \left(S_{\mathfrak{q}}\right)=\min \left[\rho\left(O^{\prime} A^{\prime}\right), \rho\left(O^{\prime} B^{\prime}\right), \rho\left(O^{\prime} C^{\prime}\right), \rho\left(O^{\prime} D^{\prime}\right)\right]
$$

Since $A^{\prime} B^{\prime}$ and $C^{\prime} D^{\prime}$ are equidistant from $O^{\circ}$ and since $B^{\prime} C^{\prime}$ and $D^{\prime} A^{\prime}$ are also equidistant from $O^{\prime}$, (observation (2) above)

$$
\inf \left(S_{\tau}\right)=\min \left[\rho\left(A^{\prime} B^{\prime}\right), \rho\left(B^{\prime} C^{\prime}\right)\right]
$$

The distance $\rho$ from the origin to a general line $\alpha x+\beta y+\chi=0$ in the $x y$ - plane is given by

$$
\rho=\frac{|\chi|}{\sqrt{\alpha^{2}+\beta^{2}}}
$$

Substituting appropriate values of $\alpha, \beta$, and $\chi$ from equations (68) and (69) into equation (82) and using equations (63) and (42), we obtain

$$
\begin{aligned}
& \rho\left(A^{\prime} B^{\prime}\right)=\frac{\left|\left(p_{11} p_{22}-p_{12} p_{21}\right) \tau_{10}\right|}{\sqrt{p_{12}{ }^{2}+p_{22}{ }^{2}}}=\frac{\left|\operatorname{det} P\left(q_{2}\right)\right| \tau_{10}}{\sqrt{p_{12}{ }^{2}+p_{22}{ }^{2}}} \\
& \rho\left(B^{\prime} C^{\prime}\right)=\frac{\left|\left(p_{11} p_{22}-p_{12} p_{21}\right) \tau_{20}\right|}{\sqrt{p_{11}{ }^{2}+p_{21}{ }^{2}}}=\frac{\left|\operatorname{det} P\left(q_{2}\right)\right| \tau_{20}}{\sqrt{p_{11}{ }^{2}+p_{21}{ }^{2}}}
\end{aligned}
$$

Substituting (83) and (84) into (81), we obtain

$$
\inf \left(s_{\tau}\right)=\min \left(\frac{\mid \operatorname{det} \mathbf{P}\left(q_{2}\right) \tau_{10}}{\sqrt{p_{12}{ }^{2}+p_{22}{ }^{2}}} \frac{\mid \operatorname{det} \mathbf{P}\left(q_{2}\right) \tau_{20}}{\sqrt{p_{11}{ }^{2}+p_{21}{ }^{2}}}\right)
$$

The $\operatorname{det} P\left(q_{2}\right)$ vanishes at $q_{2}=0, \pi$. Therefore inf $\left(S_{\tau}\right)=0$ at $q_{2}=0, \pi$.

Since the area transformed by the linear mapping $A$ is

$$
\int_{-\tau_{10}}^{\tau_{10}} \int_{-\tau_{20}}^{\tau_{20}} \operatorname{det}(\mathbf{A}) d \tau_{1} d \tau_{2}
$$

the area of the parallelogram is obtained as

$$
\begin{aligned}
& \operatorname{det}(\mathbf{A})\left[\tau_{1 o}-\left(-\tau_{10}\right)\right]\left[\tau_{2 o}-\left(-\tau_{20}\right)\right] \\
& =\operatorname{det}\left(\mathbf{R}\left(q_{1}\right)\right) \operatorname{det}\left(\mathbf{R}\left(q_{2}\right)\right) \operatorname{det}\left(\mathbf{M}\left(q_{2}\right)\right) \operatorname{det}\left(\mathbf{D}^{-1}\left(q_{1}\right)\right) 4 \tau_{10} \tau_{2 o} \\
& =\operatorname{det}\left(\mathbf{M}\left(q_{2}\right)\right) \operatorname{det}\left(\mathbf{D}^{-1}\left(q_{1}\right)\right) 4 \tau_{1 o} \tau_{2 o} \\
& =1 \frac{l_{1} l_{2} \sin q_{2}}{\left(I_{1}+m_{1} a_{1}{ }^{2}\right)\left(I_{2}+m_{2} a_{2}{ }^{2}\right)+\left(I_{2}+m_{2} a_{2}{ }^{2} \sin ^{2} q_{2}\right) m_{2} l_{1}{ }^{2}} \mid 4 \tau_{1 o} \tau_{2 o}
\end{aligned}
$$

-Property 2: $q_{1}$ - dependence of $S_{\tau}$

The supremum, infimum, and area of the set $S_{\tau}$ is independent of the joint variable $q_{1}$. For two manipulator positions $\left(q_{1}, q_{2}\right)$ and $\left(q_{1}+\phi, q_{2}\right)$,

$$
S_{\tau}\left(q_{1}+\phi, q_{2}\right)=\mathbf{R}(\phi) S_{\tau}\left(q_{1}, q_{2}\right)
$$

-Proof:

$S_{\tau}$ is a linear mapped set between actuator torques and the end-effector accelerations. So, property 2 is proved if the vertices of $S_{\tau}\left(q_{1}+\phi, q_{2}\right)$ are the simple rotation of $S_{\tau}\left(q_{1}, q_{2}\right)$. Components of vertex $A$ are

$$
\begin{aligned}
a_{11} \tau_{10}+a_{12} \tau_{20} & =\left(\cos q_{1} p_{11}-\sin q_{1} p_{21}\right) \tau_{10}+\left(\cos q_{1} p_{12}-\sin q_{1} p_{22}\right) \tau_{20} \\
& =\cos q_{1}\left(p_{11} \tau_{10}+p_{12} \tau_{20}\right)-\sin q_{1}\left(p_{21} \tau_{10}+p_{22} 20\right) \\
a_{21} \tau_{10}+a_{22} \tau_{20} & =\left(\sin q_{1} p_{11}+\cos q_{1} p_{21}\right) \tau_{10}+\left(\sin q_{1} p_{12}+\cos q_{1} p_{22}\right) \tau_{20}
\end{aligned}
$$




$$
=\sin q_{1}\left(p_{11} \tau_{10}+p_{12} \tau_{20}\right)+\cos q_{1}\left(p_{21} \tau_{10}+p_{22} 20\right)
$$

Rewriting equations (92) and (93),

$$
\left[\begin{array}{lll}
a_{11} \tau_{10}+a_{12} \tau_{20} & a_{21} \tau_{10}+a_{22} \tau_{20}
\end{array}\right]^{T}=\mathbf{R}(\phi) \quad\left[p_{11} \tau_{10}+p_{12} \tau_{2 o} \quad p_{21} \tau_{10}+p_{22} \tau_{20}\right]^{T}
$$

Similarly, other vertices of $S\left(q_{1}+\phi, q_{2}\right)$ can be shown as a product of $R(\phi)$ and $s_{l}\left(q_{1}, q_{2}\right)$. Therefore,

$$
s_{\tau}\left(q_{1}+\phi, q_{2}\right)=\mathbf{R}(\phi) s_{\tau}\left(q_{1}, q_{2}\right)
$$

-Property $3: q_{2}$ - dependence of $s_{\tau}$

The supremum, infimum, and area of the set $s_{\tau}$ depends only on the joint variable $q_{2}$.

We merely state this property to emphasize that the size of $s_{\tau}$ depends only on $q_{2}$, a property which is to be expected since, everything else being the same, two positions for which $q_{2}$ is identical [i.e. $\left(q_{1}, q_{2}\right)$ and $\left.\left(q_{1}^{\prime}, q_{2}\right)\right]$ are equivalent from a kinematic and dynamic standpoint. The property follows obviously from the equations (72)-(74) which depend only on $q_{2}$. 


\section{Quadratic mapping}

For the given (constraint) set $\mathrm{F}$ of allowable rates-of-change, described by (10)and represented by (the interior and the boundary of) the rectangle $E_{1} F_{1} E_{2} F_{2}$ shown in Figure 6-a, we defined the set $s_{\dot{q}}$ of all allowable $\alpha_{\dot{q}}$ in section 2 as

$$
s_{\dot{q}}=\left\{\alpha_{\dot{q}} \mid(\exists \dot{q} \in F)\left(\alpha_{\dot{q}}=B(\dot{q}\}^{2}\right)\right\}
$$

As in the previous section, the following questions are important

1. How is $\mathrm{S}_{\dot{q}}$ described?

2. What is the size of $s_{\dot{q}}$ ? Specifically, what is its infimum and supremum ?

3. How does $s_{\dot{q}}$ depend on the joint-variables $q_{1}$ and $q_{2}$.

\subsection{Description of $S_{\dot{q}}$}

If we define a vector

$$
\mathbf{y}=\left[\begin{array}{ll}
y_{1} & y_{2}
\end{array}\right]^{T}=\left[\dot{q}_{1}^{2}\left(\dot{q}_{1}+\dot{q}_{2}\right)^{2}-\dot{q}_{1}^{2}\right]^{T}
$$

then equation (32) can be expressed as

$$
\alpha_{\dot{q}}=\mathbf{B}(\mathbf{q}) \mathbf{y}
$$

Therefore the mapping (32) can be viewed as the product of the quadratic mapping (95) from the $\dot{\mathbf{q}}$ - plane to $\mathbf{y}$ plane followed by the linear mapping (96) from the $y$-plane to the $\alpha_{\dot{q}}$ plane.

The quadratic mapping (95) maps the constraint set $\mathrm{F}$ in the $\dot{\mathbf{q}}$ - plane into a set in the $\mathbf{y}$ - plane which we denote by $s_{\mathbf{y}}$. Then the linear mapping (96) maps this set $S_{\mathbf{y}}$ into a set in the $\alpha_{\dot{q}}$ plane which is simply the set $s_{\dot{q}}$ defined in (35).

We will therefore first obtain the set $s_{j}$ from the constraint set $F$ under the quadratic mapping (95). $S_{\dot{q}}$ is then determined from $S_{y}$ under the linear mapping (96).

\subsubsection{The quadratic map and the description of $S_{y}$}

Formally, we define $s_{y}$ as follows:

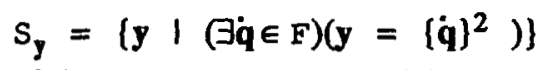

Using equation (21) we can write (95) explicitly as

$$
\begin{aligned}
& y_{1}=\dot{q}_{1}^{2} \\
& y_{2}=\left(\dot{q}_{1}+\dot{q}_{2}\right)^{2}-\dot{q}_{1}^{2}
\end{aligned}
$$

We now have to determine the mapping of (the interior and the boundary of) the $\dot{q}$ - plane rectangle $E_{1} F_{1} E_{2} F_{2}$ into the $y$ - plane as determined by (98) and (99).

The notation

$$
X_{1} \rightarrow X
$$

will be used to denote the fact that the point $X_{1}$ in the $\dot{q}$ - plane maps into the point $X$ in the $y$ - plane, i.e. $X$ is the image of $X_{1}$. 


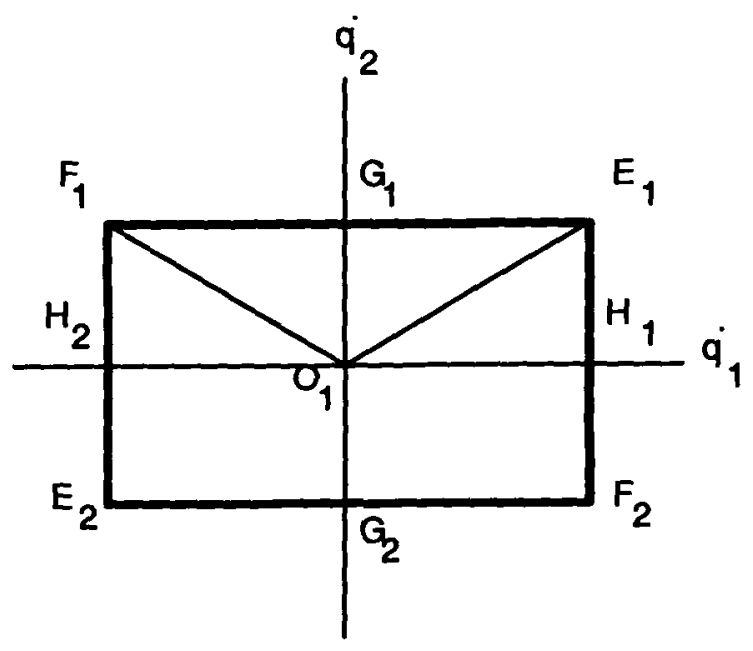

Figure 4: an available joint velocity set

From equations (98) and (99) we use that the pair of points $X_{1}\left(\dot{q}_{1}, \dot{q}_{2}\right)$ and $X_{2}\left(-\dot{q}_{1},-\dot{q}_{2}\right)$ in the $\dot{q}$ - plane both have the same image $X\left(\dot{\mathrm{q}}_{1}^{2},\left(\dot{\mathrm{q}}_{1}+\dot{\mathrm{q}}_{2}\right)^{2}-\dot{\mathrm{q}}_{1}^{2}\right)$ in the $\mathrm{y}-$ plane, i.e.

$$
x_{1}\left(\dot{q}_{1}, \dot{q}_{2}\right), x_{2}\left(-\dot{q}_{1},-\dot{q}_{2}\right) \rightarrow X\left(\dot{q}_{1}^{2},\left(\dot{q}_{1}+\dot{q}_{2}\right)^{2}-\dot{q}_{1}^{2}\right)
$$

Consider the rectangle $E_{1} F_{1} E_{2} F_{2}$ in Figure 4. A consequence of (101) is that the quadrants $O_{1} H_{1} E_{1} G_{1}$ and $\mathrm{O}_{1} \mathrm{H}_{2} \mathrm{E}_{2} \mathrm{G}_{2}$ of $\mathrm{E}_{1} \mathrm{~F}_{1} \mathrm{E}_{2} \mathrm{~F}_{2}$ both map into the same region of the $\mathrm{y}$ - plane; Similarly the quadrants $\mathrm{O}_{1} \mathrm{G}_{1} \mathrm{~F}_{1} \mathrm{H}_{2}$ and $\mathrm{O}_{1} \mathrm{G}_{2} \mathrm{~F}_{2} \mathrm{H}_{1}$ ( of $\mathrm{E}_{1} \mathrm{~F}_{1} \mathrm{E}_{2} \mathrm{~F}_{2}$ ) both map into the same region of the $\mathrm{y}$ - plane. Therefore we only need to determine the region of the $y$ - plane into which the "upper-half" $\mathrm{H}_{1} \mathrm{E}_{1} \mathrm{~F}_{1} \mathrm{H}_{2}$ (of the rectangle $\mathrm{E}_{1} \mathrm{~F}_{1} \mathrm{E}_{2} \mathrm{~F}_{2}$ ) maps. Formally $\mathrm{H}_{1} \mathrm{E}_{1} \mathrm{~F}_{1} \mathrm{H}_{2}$ is described:

$$
F^{\prime}=\left\{\dot{\mathbf{q}} \mid(\exists \dot{\mathbf{q}} \in \mathbf{F})\left(\ddot{q}_{2} \geq 0\right)\right\}
$$

The required set $S_{y}$ is therefore the image of $F^{\prime}$ under the quadratic mapping (98) and (99). To determine $S_{y}$ we first need to establish the following:

1. the image of the points $\mathrm{O}_{1}, \mathrm{H}_{1}, \mathrm{E}_{1}, \mathrm{G}_{1}, \mathrm{~F}_{1}$, and $\mathrm{H}_{2}$ under the mapping (98) and (99).

2. the image of a line

$$
\dot{q}_{2}=\dot{m} \dot{q}_{1}
$$

of slope $m$ passing through the origin $O_{1}$.

If $\mathrm{O}, \mathrm{H}, \mathrm{E}, \mathrm{G}, \mathrm{F}$ denote the image of points $\mathrm{O}_{1}, \mathrm{H}_{1}, \mathrm{E}_{1}, \mathrm{G}_{1}$, and $\mathrm{F}_{1}$, then from (98) and (99) we can write

$$
\begin{aligned}
& O_{1}(0,0) \rightarrow O(0,0) \\
& H_{1}\left(\dot{q}_{10}, 0\right), H_{2}\left(-\dot{q}_{10}, 0\right) \rightarrow H\left(\dot{q}_{10}^{2}, 0\right)
\end{aligned}
$$




$$
\begin{aligned}
& E_{1}\left(\dot{q}_{1 o}, \dot{q}_{20}\right) \rightarrow E\left(\dot{q}_{10}^{2}\left(\dot{q}_{10}+\dot{q}_{20}\right)^{2}-\dot{q}_{10}{ }^{2}\right) \\
& F_{1}\left(-\dot{q}_{1 o}, \dot{q}_{20}\right) \rightarrow F\left(\dot{q}_{10}^{2}\left(\dot{q}_{10}-\dot{q}_{20}\right)^{2}-\dot{q}_{10}{ }^{2}\right) \\
& G_{1}\left(0, \dot{q}_{20}\right) \rightarrow G\left(0, \dot{q}_{20}^{2}\right)
\end{aligned}
$$

Note that the points $\mathrm{H}_{1}$ and $\mathrm{H}_{2}$ have the same image as is to be expected from (101). Also, the origin $\mathrm{O}_{1}$ of the $\dot{\mathrm{q}}$ plane maps into the origin $O$ of the $y$ - plane. Using (98) and (99), the line (103) in the $y$ - plane maps into the set of points

$$
\begin{aligned}
& y_{1}=\dot{q}_{10}{ }^{2} \\
& y_{2}=\left(\dot{q}_{1}+m \dot{q}_{1}\right)^{2}-\dot{q}_{1}{ }^{2}=\dot{q}_{1}^{2}\left(m^{2}+2 m\right)
\end{aligned}
$$

Equations (109) and (110) are the parametric equations of the straight line

$$
y_{2}=\left(m^{2}+2 m\right) y_{1} \text {. }
$$

Therefore a line passing through the origin and of slope $m$ in the $\dot{q}$ - plane maps into a line passing through the origin and of slope $\left(m^{2}+2 m\right)$ in the $y$ - plane.

To obtain the image in the $y$ - plane of the rectangle $\mathrm{H}_{1} \mathrm{E}_{1} \mathrm{~F}_{1} \mathrm{H}_{2}$, it is convenient to divide $\mathrm{H}_{1} \mathrm{E}_{1} \mathrm{~F}_{1} \mathrm{H}_{2}$ into four triangular sections $\mathrm{O}_{1} \mathrm{H}_{1} \mathrm{E}_{1}, \mathrm{O}_{1} \mathrm{E}_{1} \mathrm{G}_{1}, \mathrm{O}_{1} \mathrm{G}_{1} \mathrm{~F}_{1}$, and $\mathrm{O}_{1} \mathrm{~F}_{1} \mathrm{H}_{2}$ and separately determine the image set for each of these sections. The required image set is simply the union of the four image sets.

In order to determine its image set, it is convenient to think of each triangular section as composed of line segments passing through the origin. This will enable us to readily determine the interior of the image set.

\section{Image set of $\mathrm{O}_{1} \mathrm{H}_{1} \mathrm{E}_{1}$ :}

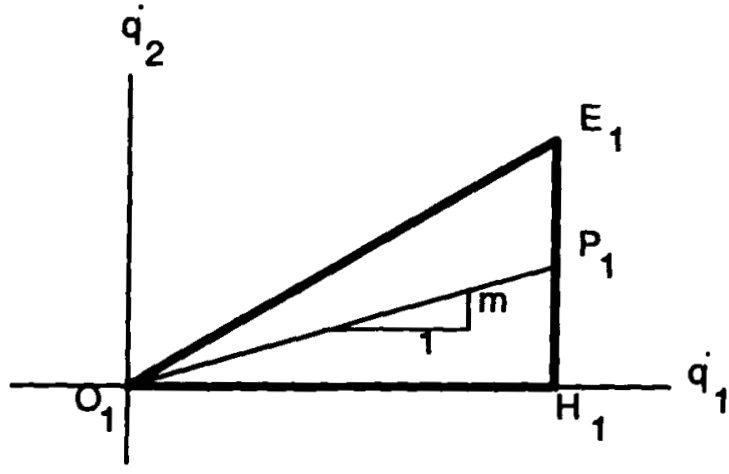

(a)

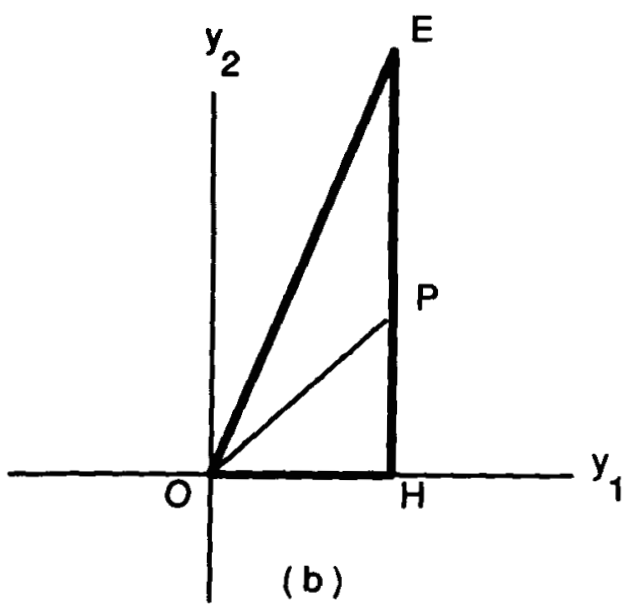

Figure 5: Image set of $\mathrm{O}_{1} \mathrm{H}_{1} \mathrm{E}_{1}$ 
Let $\left(S_{y}\right)_{1}$ denote the image set of the triangle $\mathrm{O}_{1} \mathrm{H}_{1} \mathrm{E}_{1}$ (see Figure 5-a ). Since a line passing through the origin $\mathrm{O}_{1}$ in the $\dot{q}$ plane maps into a line passing through the origin of the $y$-plane described by equation (103), the image of the generic line segment $O_{1} P_{1}$ of slope $m$ passing through $O_{1}$, shown in Figure $5-a$, will be a line segment of slope $\left(\mathrm{m}^{2}+2 \mathrm{~m}\right)$ passing through the origin $O$ in the $y$-plane. We now only need to determine the images of the end points $O_{1}(0,0)$ and $P_{1}\left(\dot{q}_{1 o}, \dot{q}_{2}\right)$ of the $O_{1} P_{1}$. The image of $O_{1}$, is of course, (see equation (104). ), the origin $O$ of the $y$ - plane. Let $P$ denote the image of $P_{1}$. Then the line segment $O_{1} P_{1}$ maps into the line segment $O P$ with one end-point at the origin $O$ of the $y$ - plane. All we need to do now is to determine the locus of $P$ as $P_{1}$ moves along the line segment $H_{1} E_{1}$. Using equations (98) and (99), the image of the line segment $H_{1} E_{1}$ described by the quations

$$
\dot{q}_{1}=\dot{q}_{10},\left(0 \leq \dot{q}_{2} \leq \dot{q}_{20}\right)
$$

is the line segment $\mathrm{HE}$, described by the equation

$$
y_{1}=\dot{q}_{10}^{2},\left(0 \leq y_{2} \leq\left(\dot{q}_{10}+\dot{q}_{20}\right)^{2}-\dot{q}_{10}{ }^{2}\right) \text {. }
$$

Furthermore the images $H$ and $E$, respectively, of points $H_{1}$ and $E_{1}$ are given by equations (105) and (106).

Therefore

1. the locus of $P$ in the $y$ - plane is the line segment $\mathrm{HE}$,

2. the several line $O_{1} P_{1}$ of slope $m$ maps into the line $O P$ passing through the origin $O$ whose equation is given by (111) and whose end-point $P$ lies on the line segment and

3. any point on $\mathrm{O}_{1} \mathrm{P}_{1}$ maps into a point on $\mathrm{OP}$.

Therefore the image $\left(\mathrm{S}_{\mathrm{y}}\right)_{1}$ of the (interior and boundary of the) $\mathrm{O}_{1} \mathrm{H}_{1} \mathrm{E}_{1}$ is the (interior and boundary of the) triangle $\mathrm{OHE}$, shown in Figure 5-b whose vertices $\mathrm{O}, \mathrm{H}, \mathrm{E}$ are given, respectively, by equations (104), (105), and (106). ( $O$ is of course the origin of the $y$-plane!).

\section{Image-Set of $\mathbf{O}_{1} \mathbf{E}_{1} \mathbf{G}_{1}$}

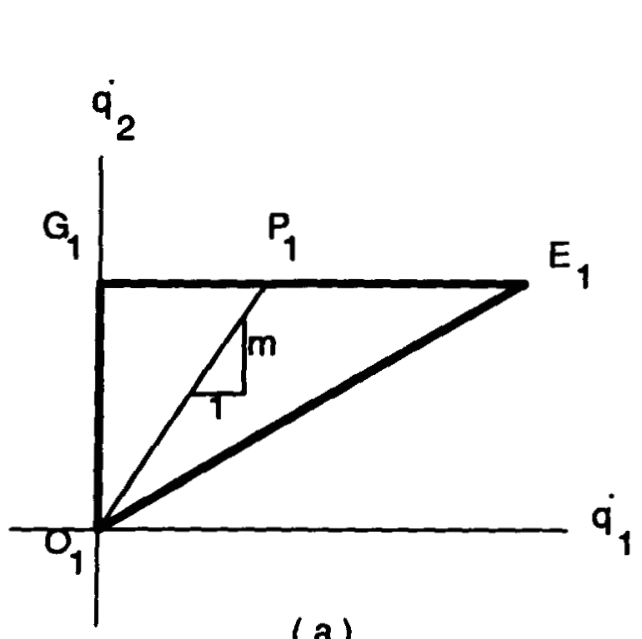

(a)

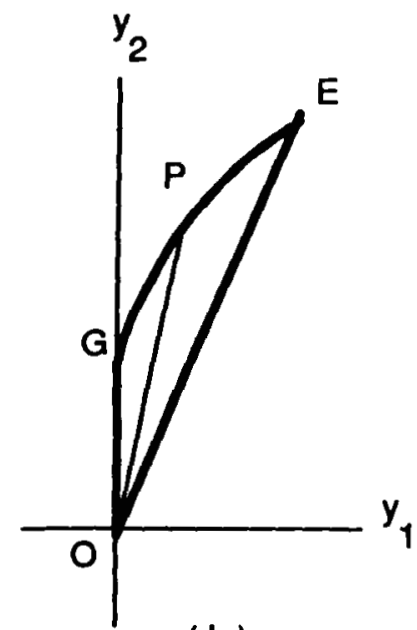

(b)

Figure 6: Image set of $\mathrm{O}_{1} \mathrm{E}_{1} \mathrm{G}_{1}$ 
Let $\left(\mathrm{S}_{\mathrm{y}}\right)_{2}$ denote the image-set of the triangle $\mathrm{O}_{1} \mathrm{E}_{1} \mathrm{G}_{1}$, shown in Figure 6-a. Using similar arguments as above, the generic line segment $O_{1} P_{1}$ of slope $m$, shown in Figure 6-a map into a line segment of slope $\left(\mathrm{m}^{2}+2 \mathrm{~m}\right)$. If $P$ denotes the image of $P_{1}$, then the image $\left(S_{y}\right)_{2}$ now reduces to obtaining the locus of $P$ as $P_{1}$ moves along $G_{1} E_{1}$ in Figure 6-a.

From equations (98) and (99) the image of the line segment $G_{1} E_{1}$ described by the equation

$$
\dot{q}_{2}=\dot{q}_{20},\left(0 \leq \dot{q}_{1} \leq \dot{q}_{10}\right)
$$

is the parabolic segment GE in the $y$ - plane described by the equation,

and shown in Figure 6-b.

$$
\left(y_{2}-\dot{q}_{20}{ }^{2}\right)^{2}=4 \dot{q}_{20}{ }^{2} y_{1},\left(\dot{q}_{10}^{2} \leq y_{2} \leq\left(\dot{q}_{10}+\dot{q}_{20}\right)^{2}-\dot{q}_{10}{ }^{2}\right) \text {. }
$$

Therefore the locus of $P$ in the $y$ - plane is the parabolic segment $G E$, the coordinates of whose end points $G$ and $E$ are given by equations (108) and (106).

The image $\left(S_{y}\right)_{2}$ of the (interior and boundary of the) triangle $O_{1} E_{1} G_{1}$ is the region OEG shown in Figure 6-b, whose vertices $\mathrm{O}, \mathrm{E}$ and $\mathrm{G}$ are given, respectively, by equations (104), (106), and (108); EG is a parabolic segment described by equation (115).

The Image-Set of $O_{1} G_{1} F_{1}$
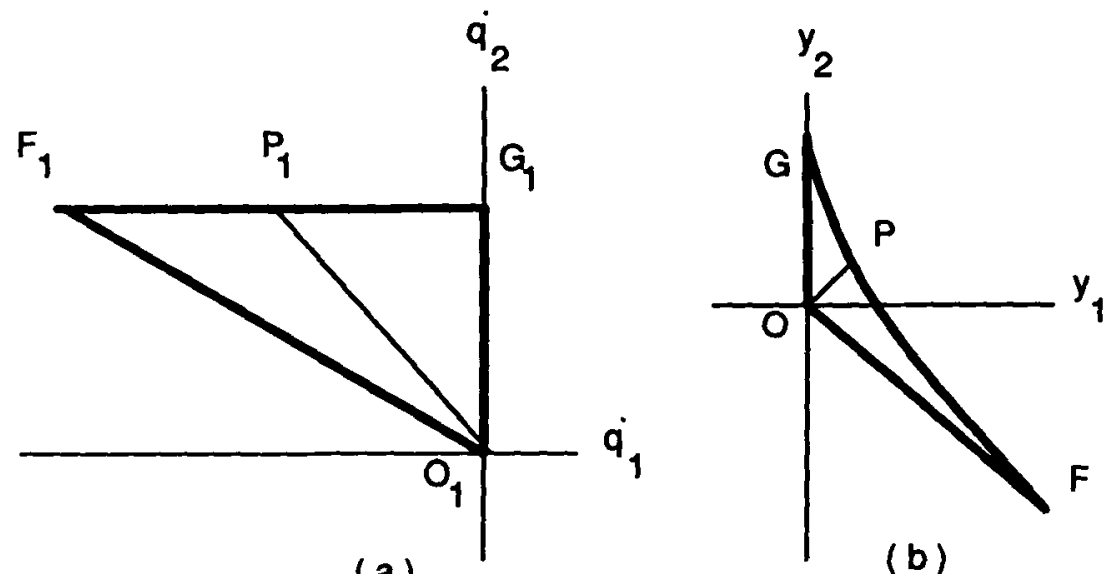

(a)

(b)

Figure 7: Image set of $\mathrm{O}_{1} \mathrm{E}_{1} \mathrm{G}_{1}$

Let $\left(S_{y}\right)_{3}$ denote the image-set of $O_{1} G_{1} F_{1}$.

As before the generic-line $O_{1} P_{1}$ incise $O_{1} G_{1} F_{1}$ ( see Figure 7-a. ) maps into the line segment $O P$, where $P$ is the image of $P_{1}$. 
In this case, we have to find the locus of $P$ as $P_{1}$ moves along $G_{1} F_{1}$.

Using equations (98) and (99), the image of the line segment $G_{1} F_{1}$, described by the equation.

$$
\dot{q}_{2}=\dot{q}_{20},\left(-\dot{q}_{10} \leq \dot{q}_{1} \leq 0\right)
$$

is the parabolic segment GF in the $y$ - plane described by the equations,

$$
\left(y_{2}-\dot{q}_{20}^{2}\right)^{2}=4 \dot{q}_{20}^{2} y_{1},\left(\left(\dot{q}_{10}-\dot{q}_{20}\right)^{2}-\dot{q}_{10}{ }^{2} \leq y_{2} \leq \dot{q}_{10}{ }^{2}\right) \text {. }
$$

Therefore the locus of $P$ in the $y$ - plane is the parabolic segment GF, the coordinates of whose end-points $G$ and $F$ are given by equations (108) and (107).

The image $\left(S_{y}\right)_{3}$ of the (interior and boundary of the) triangular segment $O_{1} G_{1} F_{1}$ is the region OGF shown in Figure 7-b, whose vertices $O, G$, and $F$ are given, respectively, by equations (104), (108) and (107). Note that $\left(\mathrm{S}_{\mathrm{y}}\right)_{3}$ is not convex.

\section{Image-Set of $\mathrm{O}_{1} \mathrm{~F}_{1} \mathrm{H}_{2}$}

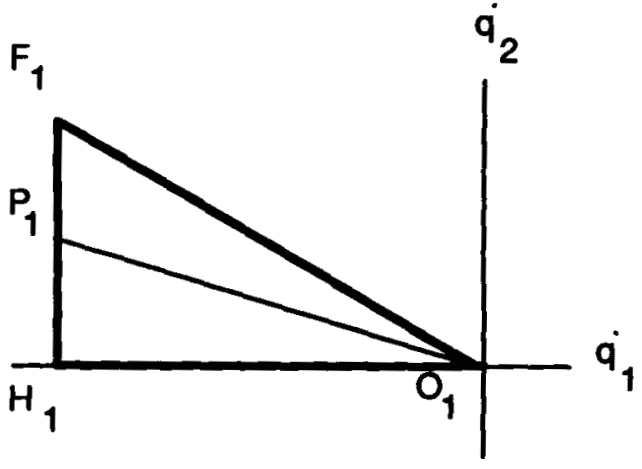

(a)

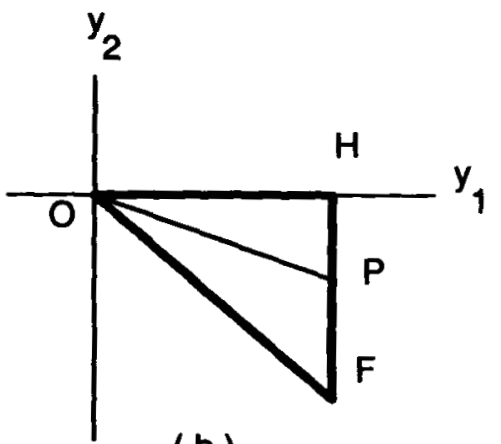

(b)

Figure 8: Image set of $\mathrm{O}_{1} \mathrm{~F}_{1} \mathrm{H}_{2}$

Let $\left(\mathrm{S}_{\mathrm{y}}\right)_{4}$ denote the image-set of the triangle $\mathrm{O}_{1} \mathrm{~F}_{1} \mathrm{H}_{2}$ in Figure $10-\mathrm{a}$.

Then the procedure for finding $\left(\mathrm{S}_{\mathrm{y}}\right)_{4}$ of the (interior and boundary of the ) triangle $\mathrm{O}_{1} \mathrm{~F}_{1} \mathrm{H}_{2}$ (shown in Figure 8-a ) is the triangle OFH, shown in Figure 8-b, whose vertices $\mathrm{O}, \mathrm{F}$, and $\mathrm{H}$ are given by equations (104), (107) and (105).

Image-Set of $H_{1} E_{1} F_{1} H_{2}$

The image-set $S_{y}$ of the rectangle $\mathrm{H}_{1} \mathrm{E}_{1} \mathrm{~F}_{1} \mathrm{H}_{2}$ is given by

$$
s_{y}=U_{i=1,2,3,4}\left(s_{y}\right)_{i} \text {. }
$$

$S_{y}$ is shown in Figure 9.

The image-set $S_{y}$ of $F$ is the (interior and boundary of the) region OGEF, shown in Figure 11, whose vertices $O, G, E$ and $F$ are given, respectively, by equations (104), (108), (106) and (107). The boundaries of OGEF are the 


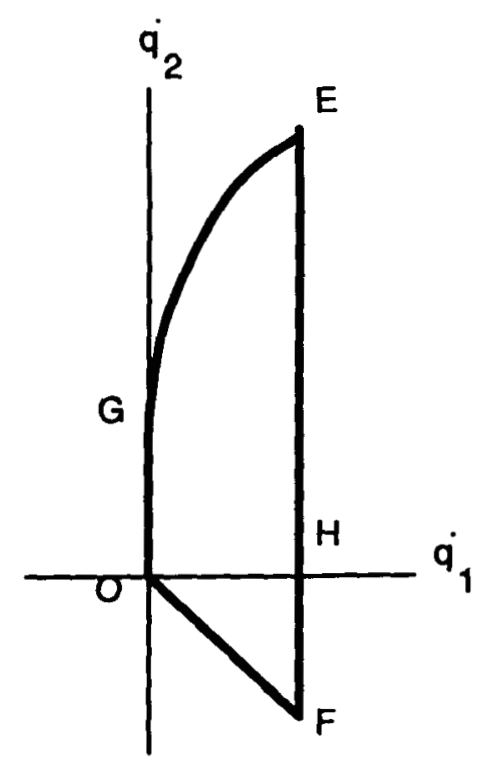

Figure 9: Image set $\mathrm{S}_{\mathrm{u}}$

line segments $O G, O F$, and $F E$ and the parabolic segment $G E$ whose equations are as follows:

$$
\begin{aligned}
& \text { OG } y_{1}=0,\left(0 \leq y_{2} \leq \dot{q}_{20}^{2}\right) \\
& \text { OF } y_{2}=\frac{\left(\dot{q}_{10}-\dot{q}_{20}\right)^{2}-\dot{q}_{10}^{2}}{\dot{q}_{10}{ }^{2}} y_{1},\left(0 \leq y_{1} \leq \dot{q}_{10}^{2}\right) \\
& \text { FE } y_{1}=\dot{q}_{10}{ }^{2},\left(\left(\dot{q}_{10}-\dot{q}_{20}\right)^{2}-\dot{q}_{10}{ }^{2} \leq y_{2} \leq\left(\dot{q}_{10}+\dot{q}_{20}\right)^{2}-\dot{q}_{10}{ }^{2}\right) \\
& \text { GE } \quad\left(y_{2}-\dot{q}_{20}{ }^{2}\right)^{2}=4 \dot{q}_{20}{ }^{2} y_{1},\left(0 \leq y_{1} \leq \dot{q}_{10}{ }^{2}\right) .
\end{aligned}
$$

Thus OGEF is completely determined. The region OGEF is convex, even though $\left(S_{y}\right)_{3}$ is non-convex. This is a consequence of the fact that the non-convex boundary of $\left(S_{y}\right)_{3}$ lies in the interior of $S_{y}$.

It will be useful in section 7 to approximate the parabolic segment EG by a straight line; consequently the region OGEF (i.e., $s_{y}$ is approximated by a quadrilateral ). Two approximations, shown in Figure 12, which are of interest in the sequel are the following:

1. the parabolic segment EG is approximated by the straight line segment EG joining E and G; the quadrilateral OGEF which is the corresponding approximation to the region OGEF, $\left(\mathrm{S}_{\mathrm{y}}\right)$, will be called the inner approximation to $S_{\mathbf{y}}$.

2. the parabolic segment EG is approximated by the line segment EI which is tangent to the parabolic segment at $E$ and which intersects the $y_{2}$-axis at $I$. The quadrilateral OIEF which is the corresponding approximation to the region OGEF, $\left(S_{y}\right)$, will be called the outer approximation to $S_{y}$.

The coordinate of I are given by 


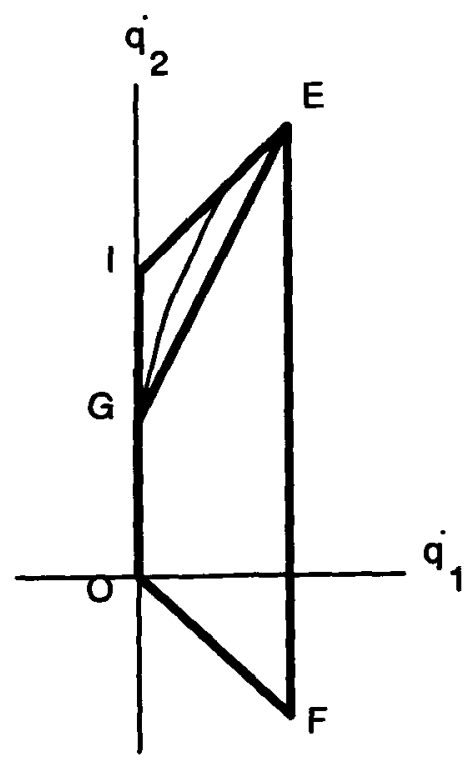

Figure 10: Approximation of Image set

$$
I\left(0,2 \dot{q}_{20}^{2}\right) \text {. }
$$

\subsubsection{The linear mapping (96) and the determination of $S_{\dot{q}}$}

From equation (35), the desired image set $s_{\dot{q}}$ is the image of $s_{y}$ under the linear mapping (96). The matrix B(q) which characterize the linear mapping (96) is given by equation (27). Combining equations (26) and (27) and using the decompositions of the $\mathbf{J}(q)$ and $\mathbf{E}(q)$ matrices given, respectively, in equations (58) and (59), we can write $B(q)$ as

$$
\mathbf{B}(\mathbf{q})=\mathbf{R}\left(q_{1}\right) \mathbf{R}\left(q_{2}\right)\left\{\begin{array}{lll}
-\mathbf{M}\left(q_{2}\right) & \mathbf{D}^{-1}\left(q_{2}\right) \mathbf{V}\left(q_{2}\right)-\mathbf{N}\left(q_{2}\right)
\end{array}\right)
$$

Defining the matrix $\mathbf{S}\left(\mathrm{q}_{2}\right)$,

$$
\mathbf{S}\left(q_{2}\right)=\mathbf{R}\left(q_{2}\right)\left\{-\mathbf{M}\left(q_{2}\right) \mathbf{D}^{-1}\left(q_{2}\right) \mathbf{V}\left(q_{2}\right)-\mathbf{N}\left(q_{2}\right)\right\},
$$

we can write $\mathbf{B}(\mathbf{q})$ as

$$
\mathbf{B}(\mathbf{q})=\mathbf{R}\left(q_{1}\right) \mathbf{S}\left(q_{2}\right) .
$$

Therefore $B(q)$ can be written as the product of a matrix $\mathbf{S}$ which is a function of $q_{2}$ only and a simple orthogonal matrix which depends on $q_{1}$ only.

The $(i, j)$ element of the matrices $S\left(q_{2}\right)$ and $B(q)$ will be denoted, respectively, by $s_{i j}$ and $b_{i j}$.

If $O^{\prime}, G^{\prime}, E^{\prime}, F^{\prime}$, and $I^{\prime}$ denote, respectively, the images in the $A_{\dot{q}}-$ plane of $O, G, E, F$, and $I$, then using (96) we can write the following: 


$$
\begin{aligned}
& E\left(\dot{q}_{1 o}{ }^{2},\left(\dot{q}_{1 o}+\dot{q}_{2 o}\right)^{2}-\dot{q}_{1 o}{ }^{2}\right) \rightarrow E^{\prime}\left(\left(b_{11}-b_{12}\right) \dot{q}_{1 o}{ }^{2}+b_{12}\left(\dot{q}_{1 o}+\dot{q}_{2 o}\right)^{2},\left(b_{21}-b_{22}\right) \dot{q}_{10}{ }^{2}+b_{22}\left(\dot{q}_{1 o}+\dot{q}_{2 o}\right)^{2}\right) \\
& F\left(\dot{q}_{1 o}{ }^{2},\left(\dot{q}_{10}-\dot{q}_{20}\right)^{2}-\dot{q}_{10}{ }^{2}\right) \rightarrow F^{\prime}\left(\left(b_{11}-b_{12}\right) \dot{q}_{1 o}{ }^{2}+b_{12}\left(\dot{q}_{1 o}-\dot{q}_{20}\right)^{2},\left(b_{21}-b_{22}\right) \dot{q}_{1 o}{ }^{2}+b_{22}\left(\dot{q}_{10}-\dot{q}_{20}\right)^{2}\right) \\
& 0(0,0) \rightarrow 0^{\prime}(0,0) \\
& G\left(0, \dot{q}_{20}^{2}\right) \rightarrow G^{\prime}\left(b_{12} \dot{q}_{2 o}^{2}, b_{22} \dot{q}_{2 o}^{2}\right)
\end{aligned}
$$

The images $E^{\prime} F^{\prime}, O^{\prime} E^{\prime}, O^{\prime} F^{\prime}$, and $O^{\prime} G^{\prime}$ of the linesegments $E F, O E, O F$, and $O G$ are line segments described by the following equations:

$$
\begin{aligned}
& E^{\prime} F^{\prime} \cdot \frac{1}{b_{12}} \alpha_{1 \dot{q}}-\frac{1}{b_{22}} \alpha_{2 \dot{q}}+\left(-\frac{b_{11}}{b_{12}}+\frac{b_{21}}{b_{22}}\right) \dot{q}_{1 o}{ }^{2}=0 \\
& O^{\prime} E^{\prime} \cdot \frac{\alpha_{1 \dot{q}}}{\left(b_{11}-b_{12}\right) \dot{q}_{1 o}{ }^{2}+b_{12}\left(\dot{q}_{10}+\dot{q}_{20}\right)^{2}}=\frac{\alpha_{2 \dot{q}}}{\left(b_{21}-b_{22}\right) \dot{q}_{1 o}{ }^{2}+b_{22}\left(\dot{q}_{1 o}+\dot{q}_{20}\right)^{2}} \\
& O^{\prime} F^{\prime} \cdot \frac{\alpha_{1 \dot{q}}}{\left(b_{11}-b_{12} \dot{q}_{10}{ }^{2}+b_{12}\left(\dot{q}_{10}-\dot{q}_{20}\right)^{2}\right.}=\frac{\alpha_{2 \dot{q}}}{\left(b_{21}-b_{22}\right) \dot{q}_{1 o}{ }^{2}+b_{22}\left(\dot{q}_{1 o}-\dot{q}_{2 o}\right)^{2}} \\
& O^{\prime} G^{\prime} \cdot \frac{\alpha_{1 \dot{q}}}{b_{12}}=\frac{\alpha_{2 \dot{q}}}{b_{22}}
\end{aligned}
$$

The parabolic segment EG maps into a parabolic segment $\mathrm{G}^{\prime} \mathrm{E}^{\prime}$.

We can therefore write: The image set $S_{\dot{q}}$ of the set $F$ is the (interior and boundary) of the region E' $F^{\prime} O^{\prime} G^{\prime}$, shown in Figure 11, whose vertices $E^{\prime}, F^{\prime}, O^{\prime}$, and $G^{\prime}$ are given, respectively, by equations (127), (128), (130), and (129). The segments $E^{\prime} F^{\prime}, O^{\prime} E^{\prime}, O^{\prime} F^{\prime}$, and $O^{\prime} G^{\prime}$ are given, respectively, by equations (131), (132), (133), and (134). Thus $s_{\dot{q}}$ is completely determined.

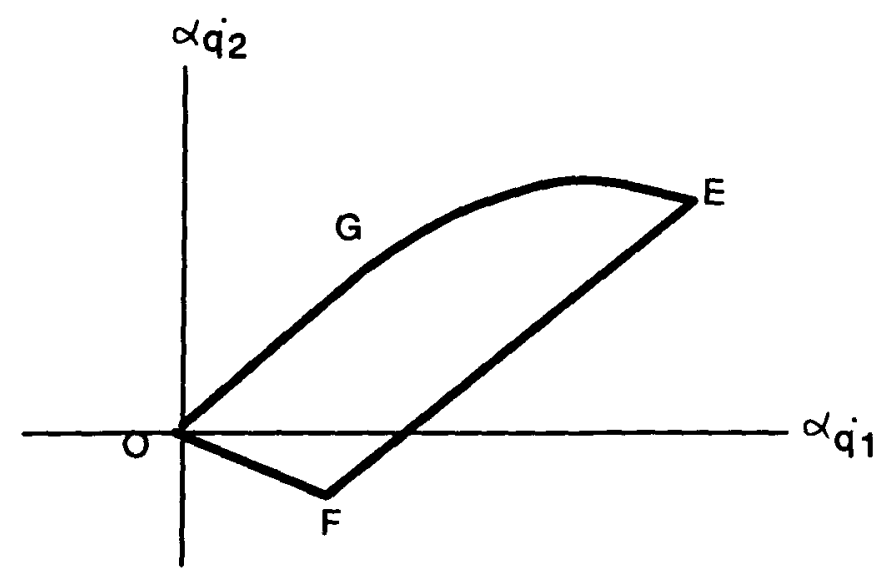

Figure 11: Image set $s_{\dot{q}}$ 


\subsection{Properties of $S_{\dot{q}}$}

In this section, we derive expressions for the supremum and infimum of $s_{\dot{q}}$.

\subsubsection{Furthest vertex of $S_{\dot{q}}$}

We first show that the furthest vertex of $S_{\dot{q}}$ is $E^{\prime}$. Inspection of equations (119) through (122) shows that the furthest vertex of the set $S_{y}$ is $E$. Since the set $S_{\dot{q}}$ is the image of $S_{y}$ under a linear mapping, the furthest vertex of $S_{\dot{q}}$ is the image of the furthest vertex, $E$, of $S_{\mathbf{y}}$. Therefore $E^{\prime}$ is the furthest vertex of $S_{\dot{q}} \cdot$

\subsubsection{Supremum of $S_{\dot{q}}$}

The supremum of $s_{\dot{q}}$ is the distance of the furthest vertex $E^{\prime}$ from the origin. Using equation (127), we obtain

$$
\begin{aligned}
& \sup \left(s_{\dot{q}}\right)= \\
& {\left[\left(s_{11}-s_{12}\right)^{2}+\left(s_{21}-s_{22}\right)^{2}\right] \dot{q}_{10}{ }^{4}+\left[s_{12}{ }^{2}+s_{22}{ }^{2}\right]\left(\dot{q}_{10}+\dot{q}_{20}\right)^{4}+2\left[s_{12}\left(s_{11}-s_{12}\right)+s_{22}\left(s_{21}-s_{22}\right)\right] \dot{q}_{10}{ }^{2}\left(\dot{q}_{10}+\dot{q}_{20}\right)^{2} .}
\end{aligned}
$$

\subsubsection{Infimum of $S_{\dot{q}}$}

Since the origin $O^{\prime}(0,0)$ is one of the vertices of $s_{\dot{q}}$, the infimum of $s_{\dot{q}}$ is zero:

$$
\inf \left(s_{\dot{q}}\right)=0
$$




\section{The state acceleration set, $S_{u}$}

We defined the acceleration set, $S_{u}$, at a specified point $u$ in the state space as follows:

Definition of $S_{u}(q, \dot{q})$ : For a given set $T$ of allowable actuator torques described by

$$
T=\left\{\tau|| \tau_{i} \mid \leq \tau_{i o}, i=1,2\right\},
$$

the acceleration set $S_{u}$ at a point $u=[q, \dot{q}]^{T}$ in the state - space is given by

$$
S_{u}(\mathbf{q}, \dot{q})=\left\{\alpha_{u} \mid(u=(\mathbf{q}, \dot{q})), \quad(\exists \tau \in T)\left(\tau_{\mathbf{u}}=\mathbf{A}(\mathbf{q}) \tau+\mathbf{k}(\mathbf{u})\right)\right\}
$$

Thus $S_{u}$ is the image of the set $T$ under the mapping (38).

\subsection{Determination of $S_{\mathbf{u}}$}

Inspection of equation (31) and (38) reveals that

$$
\alpha_{\mathbf{u}}(\mathbf{q}, \dot{q}, \tau)=\alpha_{\tau}(\mathbf{q}, \tau)+\mathbf{k}(\mathbf{q}, \dot{q})
$$

where $\alpha_{\tau}(\mathbf{q}, \tau) \in S_{\tau}(\mathbf{q}, \tau)$ and $\alpha_{u}(\mathbf{q}, \dot{\mathbf{q}}, \tau) \in S_{\mathbf{u}}(\mathbf{q}, \dot{\mathbf{q}}, \tau)$.

From (137), we see that

$$
\alpha_{u}(\mathbf{q}, \dot{q}=0, \tau)=\alpha_{\tau}(q, \tau) .
$$

Defining,

$$
I:=\quad\left[\begin{array}{lll}
1 & 0 \\
0 & 1
\end{array}\right] \text {, }
$$

and

$$
\bar{\alpha}_{u}=\alpha_{u}(q, \dot{q}=0, \tau)
$$

then

$$
\bar{\alpha}_{\mathbf{u}}=I \quad \alpha_{\tau}(q, \tau)
$$

and

$$
\alpha_{u}=\bar{\alpha}_{u}+\mathbf{k}(\mathbf{q}, \dot{q}) .
$$

If we define a set $\dot{S}_{u}$,

$$
\bar{s}_{u}=\left\{\bar{\alpha}_{u} \mid\left(\exists \alpha_{\tau} \in S_{\tau}\right)\left(\alpha_{u}=I \quad \alpha_{\tau}\right)\right\},
$$

then $\bar{S}_{u}$ is the image in the $A_{u}$ - plane of the set $\bar{S}_{\tau}$ in the $A_{\tau}$ - plane under the identity mapping (139). From (142) the desired acceleration set $S_{u}$ at a specified point $u=[q, \dot{q}]^{T}$ in the state space is the set obtained by translating $\dot{S}_{u}$ by the (constant) displacement vector $k(q, \dot{q})$. This process of generating $S_{\mathbf{u}}$ is shown in Figure 12-a, b, and $c$.

We can write $S_{u}$ in the following equivalent form:

$$
S_{u}=\left\{\alpha_{u} \mid\left(\exists \alpha_{\tau} \in S_{\tau}\right)\left(\alpha=\alpha_{\tau}+k\right)\right\}
$$

Since $S_{\tau}$ is a parallelogram $A^{\prime} B^{\prime} C^{\prime} D^{\prime}, S_{u}$ and $S_{u}$ are also parallelograms congruent to $S_{\tau}$ but lying in the $A_{u}$ - plane. The centroid of the set $S_{u}$ has coordinates $\left(\alpha_{u 1}, \alpha_{u 2}\right)$ as shown in Figure 12-c. Loosely speaking, we can say that $S_{u}$ is obtained by translating $S_{\tau}$ by $\left(\alpha_{u 1}, \alpha_{u 2}\right)$ from the origin. 


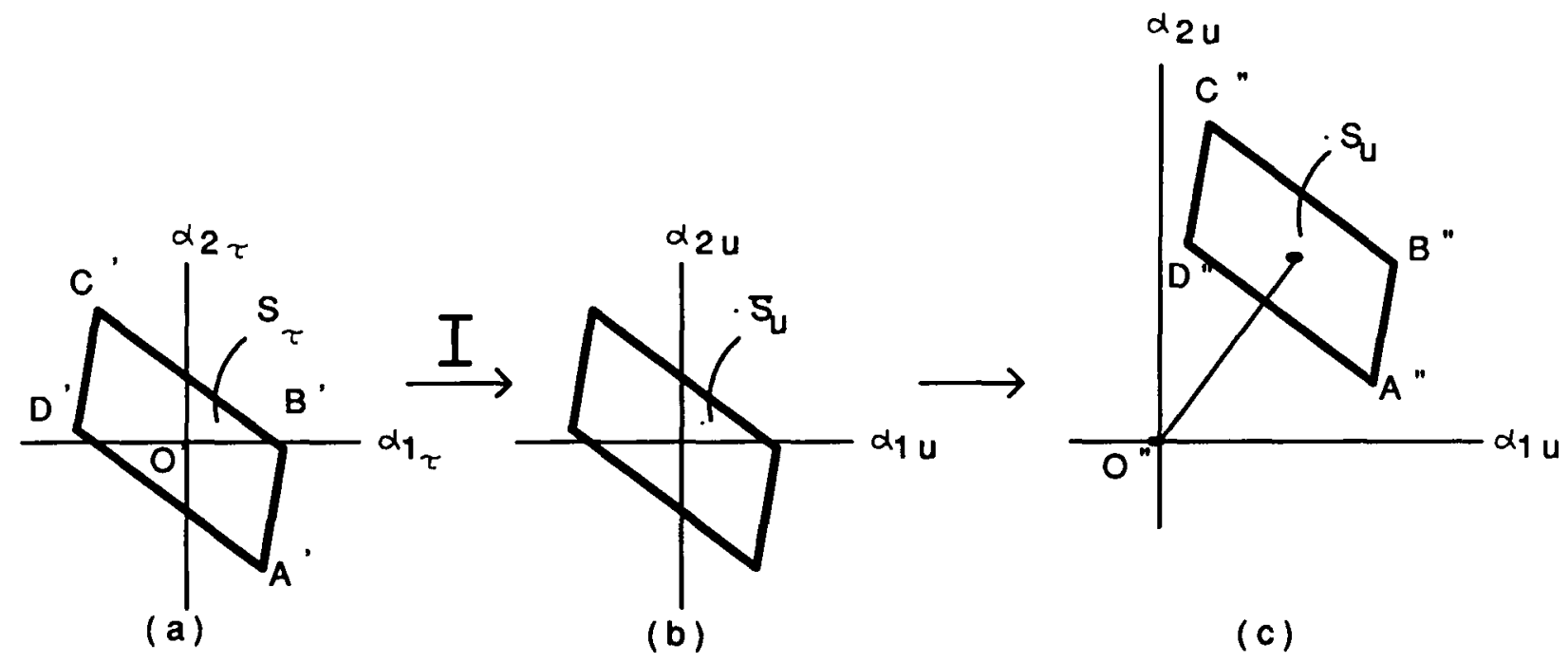

Figure 12: A state acceleration set

If $A^{\prime \prime}, B^{\prime \prime}, C^{\prime \prime}$, and $D^{\prime \prime}$ denote, respectively, the images in the $A_{u}$ - plane of points $A^{\prime}, B^{\prime}, C^{\prime}$, and $D^{\prime}$ in the $A_{\tau}$ plane (Figure 3-a), then from equations (137) and (64) through (67), we obtain

$$
\begin{aligned}
& A^{\prime}\left(a_{11} \tau_{10}+a_{12} \tau_{20}, a_{21} \tau_{10}+a_{22} \tau_{20}\right) \rightarrow A^{\prime \prime}\left(k_{1}+a_{11} \tau_{10}+a_{12} \tau_{20}, k_{2}+a_{21} \tau_{10}+a_{22} \tau_{20}\right) \\
& B^{\prime}\left(a_{11} \tau_{10}-a_{12} \tau_{20}, a_{21} \tau_{10}-a_{22} \tau_{20}\right) \rightarrow B^{\prime \prime}\left(k_{1}+a_{11} \tau_{10}-a_{12} \tau_{20}, k_{2}+a_{21} \tau_{1 o}-a_{22} \tau_{20}\right) \\
& C^{\prime}\left(-a_{11} \tau_{10}-a_{12} \tau_{20},-a_{21} \tau_{10}-a_{22} \tau_{20}\right) \rightarrow C^{\prime \prime}\left(k_{1}-a_{11} \tau_{10}-a_{12} \tau_{20}, k_{2}-a_{21} \tau_{10}-a_{22} \tau_{20}\right) \\
& D^{\prime}\left(-a_{11} \tau_{10}+a_{12} \tau_{20},-a_{21} \tau_{10}+a_{22} \tau_{20}\right) \rightarrow D^{\prime \prime}\left(k_{1}-a_{11} \tau_{10}+a_{12} \tau_{20}, k_{2}-a_{21} \tau_{10}+a_{22} \tau_{20}\right) .
\end{aligned}
$$

$S_{u}$ is the (interior and boundary) of the parallelogram $A^{\prime \prime} B^{\prime \prime} C^{\prime \prime} D^{\prime \prime}$. The sides $A^{\prime \prime} B^{\prime \prime}, B^{\prime \prime} C^{\prime \prime}, C^{\prime \prime} D^{\prime \prime}$, and $D^{\prime \prime} A "$ of the parallelogram are obtained by (137) and equations (68) through (71),

$$
\begin{aligned}
& A^{\prime \prime} B^{\prime \prime} \cdot \frac{1}{a_{12}} a_{1 \tau}-\frac{1}{a_{22}} a_{2 \tau}+\left(-\frac{a_{11}}{a_{12}}+\frac{a_{21}}{a_{22}}\right) \tau_{10}+\left(-\frac{k_{1}}{a_{12}}+\frac{k_{2}}{a_{22}}\right)=0 \\
& B^{\prime \prime} C^{\prime \prime} \cdot \frac{1}{a_{11}} a_{1 \tau}-\frac{1}{a_{21}} a_{2 \tau}+\left(-\frac{a_{12}}{a_{11}}+\frac{a_{22}}{a_{21}}\right) \tau_{20}+\left(-\frac{k_{1}}{a_{11}}+\frac{k_{2}}{a_{21}}\right)=0 \\
& C^{\prime \prime} D^{\prime \prime} \cdot \frac{1}{a_{12}} a_{1 \tau}-\frac{1}{a_{22}} a_{2 \tau}-\left(-\frac{a_{11}}{a_{12}}+\frac{a_{21}}{a_{22}}\right) \tau_{10}+\left(-\frac{k_{1}}{a_{12}}+\frac{k_{2}}{a_{22}}\right)=0 \\
& D^{\prime \prime} A^{\prime \prime} \cdot \frac{1}{a_{11}} a_{1 \tau}-\frac{1}{a_{21}} a_{2 \tau}-\left(-\frac{a_{12}}{a_{11}}+\frac{a_{22}}{a_{21}}\right) \tau_{20}+\left(-\frac{k_{1}}{a_{11}}+\frac{k_{2}}{a_{21}}\right)=0 .
\end{aligned}
$$

\subsection{Supremum of $S_{u}$}

The supremum of $S_{u}$ is a measure of the largest acceleration available (in some direction ) at a specified point in the state-space. In a similar manner to that of $S_{\tau}$, the supremum of $S_{u}$ is obtained as the distance of the furthest vertex of the parallelogram $A^{\prime \prime} B^{\prime \prime} C^{\prime \prime} D^{\prime \prime}$ from the origin $O^{\prime \prime}$ of the $A_{u}$ plane.

If $l\left(\mathrm{O}^{\prime \prime} \mathrm{A}^{\prime \prime}\right), l\left(\mathrm{O}^{\prime \prime} \mathrm{B}^{\prime \prime}\right), l\left(\mathrm{O}^{\prime \prime} \mathrm{C}^{\prime \prime}\right)$, and $l\left(\mathrm{O}^{\prime \prime} \mathrm{D}^{\prime \prime}\right)$ denote, respectively, the distances of the vertices $\mathrm{A}^{\prime \prime}, \mathrm{B}^{\prime \prime}, \mathrm{C}^{\prime \prime}$, and 
$D "$ from the origin $O^{\prime \prime}$, then from (145) through (148) we obtain

$$
\begin{aligned}
& l\left(O^{\prime \prime} A^{\prime \prime}\right)=\sqrt{\left(k_{1}+a_{11} \tau_{1 o}+a_{12} \tau_{20}\right)^{2}+\left(k_{2}+a_{21} \tau_{10}+a_{22} \tau_{20}\right)^{2}} \\
& l\left(O^{\prime \prime} B^{\prime \prime}\right)=\sqrt{\left(k_{1}+a_{11} \tau_{10}-a_{12} \tau_{20}\right)^{2}+\left(k_{2}+a_{21} \tau_{1 o}-a_{22} \tau_{20}\right)^{2}} \\
& l\left(O^{\prime \prime} C^{\prime \prime}\right)=\sqrt{\left(k_{1}-a_{11} \tau_{10}-a_{12} \tau_{20}\right)^{2}+\left(k_{2}-a_{21} \tau_{1 o}-a_{22} \tau_{20}\right)^{2}} \\
& l\left(O^{\prime \prime} D^{\prime \prime}\right)=\sqrt{\left(k_{1}-a_{11} \tau_{10}+a_{12} \tau_{20}\right)^{2}+\left(k_{2}-a_{21} \tau_{10}+a_{22} \tau_{20}\right)^{2}}
\end{aligned}
$$

The supremum of $S_{u}$ is given by

$$
\sup \left(S_{u}\right)=\max \left[l\left(O^{\prime \prime} A^{\prime \prime}\right), l\left(O^{\prime \prime} B^{\prime \prime}\right), l\left(O^{\prime \prime} C^{\prime \prime}\right), l\left(O^{\prime \prime} D^{\prime \prime}\right)\right]
$$

\subsection{Infimum of $S_{u}$}

The infimum is the maximum isotropic acceleration for a certain manipulator position in the workspace.

To obtain the infimum we must consider three cases

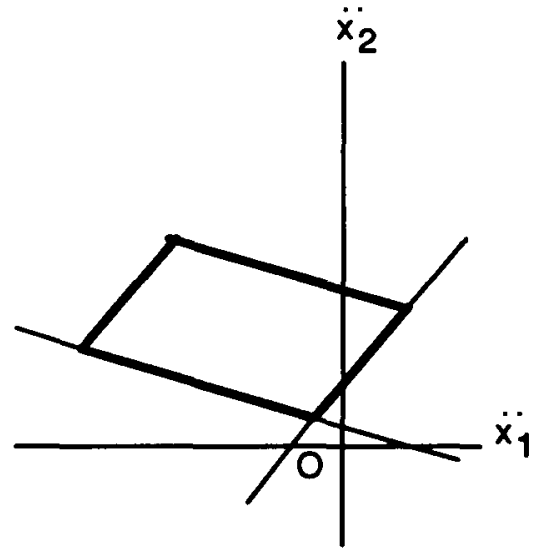

(a)

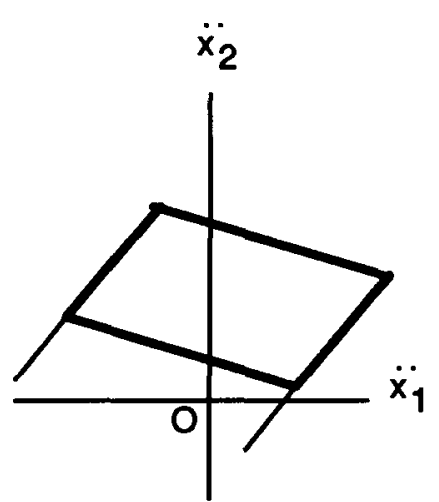

(b) and (c)

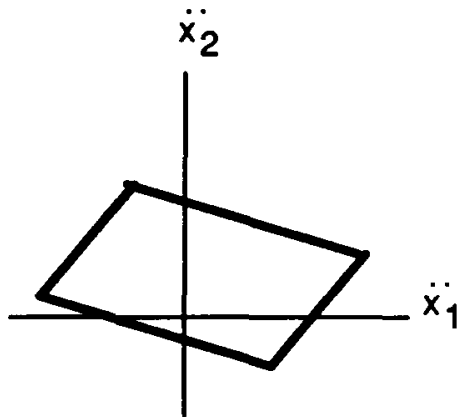

(d)

Figure 13: the relative location of a parallelogram to the origin

1. The origin $O^{\prime \prime}$ lies outside the parallelogram $A " B " C " D "$ and $O "$ does not lie between either pair of parallel lines (Figure 13-a) comprising the sides of the parallelogram.

2. The origin $O$ " lies outside the parallelogram $A " B " C " D$ " and $O^{\prime \prime}$ lies between $A " B$ " and C"D" (Figure 13-b).

3. The origin $\mathrm{O}^{\prime \prime}$ lies outside the parallelogram $\mathrm{A}^{\prime \prime} \mathrm{B} \mathrm{C}^{\prime \prime} \mathrm{D}$ " and $\mathrm{O}$ " lies between $\mathrm{B}^{\prime \prime} \mathrm{C}^{\prime \prime}$ and $\mathrm{D} \mathrm{A}^{\prime \prime}$ (Figure 13-c).

4. The origin $O^{\prime \prime}$ lies inside the parallelogram $A^{\prime \prime} B " C^{\prime \prime} D^{\prime \prime}$ (Figure 13-d).

Using well-known results from analytic geometry, the condition for $O^{\prime \prime}$ to lie between the parallel lines $A " B$ " and $C " D "$ is obtained from (149) and (151) as

condition 1: 


$$
\left(\frac{k_{1}}{a_{12}}-\frac{k_{2}}{a_{22}}\right)^{2} \leq\left(\frac{a_{11}}{a_{12}}-\frac{a_{21}}{a_{22}}\right)^{2} \tau_{1 o}^{2}
$$

the condition for $\mathrm{O}^{\prime \prime}$ to lie between the parallel lines $\mathrm{B}^{\prime \prime} \mathrm{C}^{\prime \prime}$ and $\mathrm{D}^{\prime \prime} \mathrm{A}$ " is obtained from (150) and (152) as

condition 2:

$$
\left(\frac{k_{1}}{a_{11}}-\frac{k_{2}}{a_{21}}\right)^{2} \leq\left(\frac{a_{12}}{a_{11}}-\frac{a_{22}}{a_{21}}\right)^{2} \tau_{20}^{2}
$$

Using the above two conditions, the three cases can easily be identified from the following rules:

- case 1: both conditions 1 and 2 are false.

- case 2: condition 1 is false and condition 2 is true.

- case 3: condition 1 is true and condition 2 is false.

- case 4: both conditions 1 and 2 are true.

The infimum for the three cases is obtained as follows:

case 1:(Figure 13-a)

In this case, the infimum is the distance of the closest vertex of $A " B " C " D "$ from the origin $O "$. Therefore

$$
\inf \left(S_{u}\right)=\min \left[l\left(O^{\prime \prime} A^{\prime \prime}\right), l\left(O^{\prime \prime} B^{\prime \prime}\right), l\left(O^{\prime \prime} C^{\prime \prime}\right), l\left(O^{\prime \prime} D^{\prime \prime}\right)\right]
$$

case 2:(Figure 13-b)

In this case the infimum is the distance from the origin to the nearest side, which is either $A " B "$ or $C " D "$.

let $d\left(A^{\prime \prime} B^{\prime \prime}\right)$ and $d\left(C^{\prime \prime} D^{\prime \prime}\right)$ be, respectively, the distances from $O^{\prime \prime}$ to sides $A^{\prime \prime} B^{\prime \prime}$ and $C^{\prime \prime} D^{\prime \prime}$.

$$
d\left(A^{\prime \prime} B^{\prime \prime}\right), d\left(C^{\prime \prime} D^{\prime \prime}\right)=\frac{\left|\left(a_{11} a_{22}-a_{12} a_{21}\right) \tau_{10} \pm\left(a_{22} k_{1}-a_{12} k_{2}\right)\right|}{\sqrt{a_{12}{ }^{2}+a_{22}{ }^{2}}}
$$

In a manner similar to obtaining the infimum of $S_{\tau}$, the infimum of $S_{u}$ is obtained from equations (161) as

$$
\inf \left(S_{u}\right)=\min \left[d\left(A^{n} B^{n}\right), d\left(C^{n} D^{n}\right)\right]
$$

case 3:(Figure 13-c)

The nearest side is either $B^{\prime \prime} C^{\prime \prime}$ or $D^{\prime \prime} A^{\prime \prime}$. let $d\left(B^{\prime \prime} C^{\prime \prime}\right)$ and $d\left(D^{\prime \prime} A^{\prime \prime}\right)$ be, respectively, the distances from $O^{\prime \prime}$ to sides B"C" and D"A".

$$
d\left(B^{\prime \prime} C^{\prime \prime}\right), d\left(D^{\prime \prime} A^{\prime \prime}\right)=\frac{\left|\left(a_{12} a_{21}-a_{11} a_{22}\right) \tau_{20} \pm\left(a_{21} k_{1}-a_{11} k_{2}\right)\right|}{\sqrt{a_{11}{ }^{2}+a_{21}{ }^{2}}}
$$

The infimum of $\mathrm{S}_{\mathrm{u}}$ is obtained from equations $(163)$ as

$$
\inf \left(\mathrm{S}_{\mathbf{u}}\right)=\min \left[d\left(B^{\prime \prime} C^{\prime \prime}\right), d\left(D^{\prime \prime} A^{\prime \prime}\right)\right]
$$

case 4: (Figure 13-d) The infimum is the distance from the origin to the nearest side which could be either $A^{\prime \prime} B ", B " C^{\prime \prime}, C^{\prime \prime} D^{\prime \prime}$, or $D^{\prime \prime} A^{\prime \prime}$. These distances were computed for cases 2 and 3 above. Therefore,

$$
\inf \left(S_{u}\right)=\min \left[d\left(A^{\prime \prime} B^{n}\right), d\left(B^{\prime \prime} C^{\prime \prime}\right), d\left(C^{\prime \prime} D^{n " m}, d\left(D^{\prime \prime} A^{\prime \prime}\right)\right]\right.
$$


To summarize the results of this section we can state the following lemma.

Lemma: The acceleration set $S_{u}$ at a point $u$ in the state-space of the manipulator is a parallelogram with centroid located at the point $\left(k_{1}, k_{2}\right)$ defined by equations (37); the supremum of $S_{u}$ is given by (157) and the infimum of $S_{u}$ is given by one of equations (160), (162), (164), and (165). The supremum and infimum of $S_{u}$ is independent of the joint angle $q_{1}$.

\section{Local acceleration sets}

At a given position $q=\left[q_{1}, q_{2}\right]^{T}$ in the workspace of the manipulator, we could define two sets in section 2

$$
\begin{aligned}
& \left(s_{L_{1}}=\cup_{\dot{\mathbf{q}} \in \mathrm{F}} s_{\mathbf{u}}(\mathbf{q}, \dot{\mathbf{q}})\right. \\
& \left(s_{L_{2}}=\cap_{\dot{\mathbf{q}} \in \mathrm{F}} s_{\mathbf{u}}(\mathbf{q}, \dot{\mathbf{q}})\right.
\end{aligned}
$$

The supremum of $\left(S_{L}\right)_{1}$ will be give us the magnitude of the maximum acceleration (in some direction) of the reference point $P$ at a given position $\left(q_{1}, q_{2}\right)$ of the manipulator.

The infimum of $\left(S_{L_{2}}\right.$ will give us the magnitude of the maximum acceleration of the reference point $P$ available in all direction at a given position of the manipulator. The infimum of $\left(\mathrm{S}_{L}\right)_{2}$ is called the isotropic acceleration in Khatib [1] and the local acceleration radius Kim [5].

\subsection{Determination of $\left(S_{L}\right)_{1}$}

The generic member $S_{u}$ of the set $\left(S_{L}\right)_{1}$ was described in section 6 and is shown in Figure 9. As $\dot{q}$ is varied, $S_{\dot{q}}$ is a parallelogram which moves parallel to itself. The locus of the centroid, ( $\left.k_{1}, k_{2}\right)$, of the parallelogram as $\dot{q}$ is varied is simply the boundary $O^{\prime} G^{\prime} E^{\prime} F^{\prime}$ of the set $S_{\dot{q}}$ shown in Figure 11 . Therefore we can describe $\left(S_{L}\right)_{1}$ as follows: The local acceleration set $\left(S_{L}\right)_{1}$ is the region swept out by the parallelogram $S_{u}$ as its vertex moves along the boundary O'G'E'F'. This is shown in Figure 14.

\subsection{Supremum of $\left(S_{L}\right)_{1}$}

The supremum of $\left(S_{L_{1}}\right.$ is simply the distance of the origin from the furthest point of $\left(\mathrm{S}_{L}\right)_{1}$.

To determine the furthest point of $\left(S_{L}\right)_{1}$, all we need to do is to determine

1. the furthest vertex of $O^{\prime} G^{\prime} E^{\prime} F^{\prime}$,

2. the parallelogram at the furthest vertex, and

3. the furthest vertex of this parallelogram

In section 5, we showed that $E^{\prime}$ is the furthest vertex of $O^{\prime} G^{\prime} E^{\prime} F^{\prime}$. The distances $\mathrm{d}\left(O^{\prime} A_{E^{\prime}}^{\prime}\right), \mathrm{d}\left(O^{\prime} B_{E^{\prime}}^{\prime}\right), \mathrm{d}\left(O^{\prime} C_{E^{\prime}}^{\prime}\right), \mathrm{d}($ $\left.O^{\prime} D_{E^{\prime}}^{\prime}\right)$ of the vertices of the parallelogram with centroid $E^{\prime}$ are given by (153) through (156). The supremum of $\left(\mathrm{S}_{L_{1}}\right)_{1}$ is now readily obtained as

$$
\sup \left(S_{L}\right)_{1}=\max \left[d\left(O^{\prime} A_{E^{\prime}}^{\prime}\right), d\left(O^{\prime} B_{E^{\prime}}^{\prime}\right), d\left(O^{\prime} C_{E^{\prime}}^{\prime}\right), d\left(O^{\prime} D_{E^{\prime}}^{\prime}\right)\right] \text {. }
$$


29

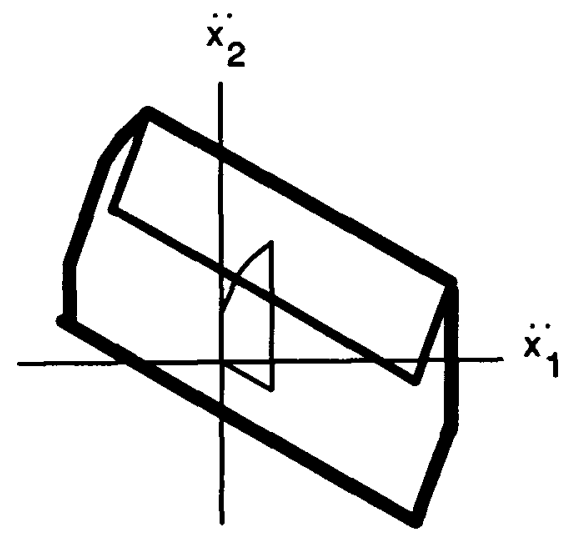

Figure 14: Determination of $\left(s_{L}\right)_{1}$

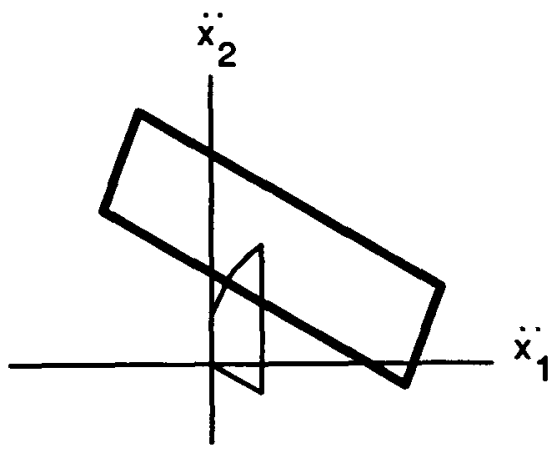

Figure 15: A local supremum

7.3 Determination of $\left(\mathrm{S}_{\mathrm{L}}\right)_{2}$

Using reasoning similar to that in the above section we can describe $\left(s_{L}\right)_{2}$ as follows: The local acceleration set $\left(S_{L}\right)_{2}$ is the largest region common to all the parallelograms generated by moving the generic parallelogram $S_{u}$ along the the boundary $O^{\prime} G^{\prime} E^{\prime} F$. 


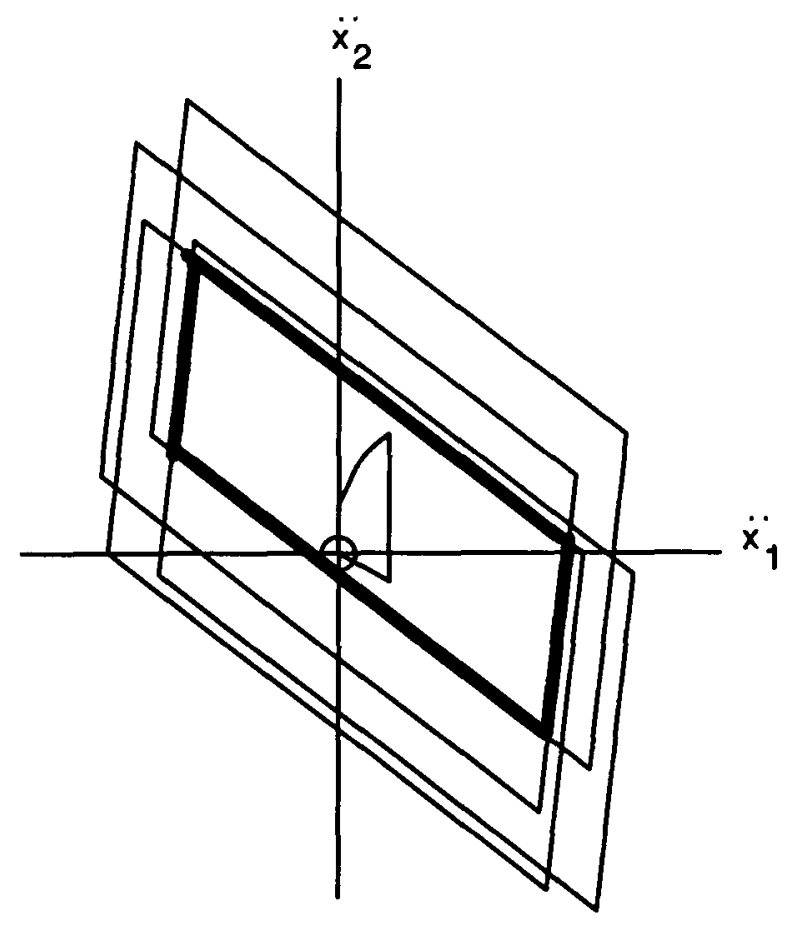

Figure 16: $\left(S_{L}\right)_{2}$ and a local infimum

\subsection{Infimum of $\left(S_{U_{2}}\right.$}

The infimum of $\left(s_{L}\right)_{2}$ is the maximum distance to the origin from the boundary of $\left(s_{L}\right)_{2}$.

To determine the infimum of $\left(S_{L}\right)_{2}$, the inner and outer approximation of the set $s_{\dot{q}}$ in section 5 (and consequently the boundary $O^{\prime} G^{\prime} E^{\prime} F^{\prime}$ ) are useful.

The problem of determining the infimum corresponding to an approximating quadrilateral reduces to examining the parallelograms with centroids at the vertices of the approximating quadrilateral since these represent the extreme parallelograms.

The procedure for finding the infimum, $r$, corresponding to an approximate quadrilateral (inner or outer) is as follows:

1. Construct the parallelogram at each of the four vertices $O^{\prime}, G^{\prime}, E^{\prime}, F^{\prime}$ or $O^{\prime}, I^{\prime}, E^{\prime}, F^{\prime}$ of the quadrilateral. Let $P_{i},(i=1,2,3,4)$ denote these four parallelograms.

2. Check if each parallelogram $P_{i}$ satisfies the two conditions (158) and (159). If all the parallelograms satisfy these conditions, then an infimum exists.

3. For each parallelogram, $P_{i}$, determine the minimum distance, $d_{i}$, from the origin to the four sides of $P_{i}$.

4. Then the infimum, $r$, of $\left(S_{L_{2}}\right.$ for the approximation is given by

$$
r=\inf \left(S_{L_{2}}=\min \left(d_{i}, i=1,2,3,4\right)\right.
$$

Let $r_{1}$ and $r_{2}$ denote, respectively, the infimum corresponding to the inner approximation OGEF and OIEF.

We now need to distinguish 3 cases.

- case 1: $r_{2}=\min \left(d_{i}, i=1,2,3,4\right)$ was obtained from the parallelogram with vertex $I$. In this case $r_{1}$ and 
$r_{2}$ are different and

$$
r_{1}<\inf \left(s_{L}\right)_{2}<r_{2}
$$

- case 2: $r_{1}=\min \left(d_{i}, i=1,2,3,4\right)$ was obtained from the parallelogram with vertex $G$. In this case $r_{1}$ and $r_{2}$ are different and

$$
r_{1}<\inf \left(\mathrm{s}_{L_{2}}<r_{2}\right.
$$

- case 3: $r_{1}$ is not obtained from the parallelogram with vertex $G$ and $r_{2}$ is not obtained from the parallelogram with vertex $I$. In this case $r_{1}$ and $r_{2}$ are both obtained from one of the other three vertices and therefore $r_{1}=r_{2}$ and

$$
\inf \left(s_{L}\right)_{2}=r_{1}=r_{2}
$$

Therefore we either obtain the inf $\left(S_{L_{2}}\right.$ exactly as in equation (170) or with tight bounds as in equations (168) or (169). 


\section{Example}

In this section, we will illustrate the manipulator dynamic properties obtained in section 4 through 7 using a two degree-of-freedom manipulator. First, we show the state accelerations at the vertices of joint velocity contribution quadrilateral in section 6 . The contribution, $\alpha_{\tau}$, of actuator torques in section 4 is the local acceleration set with the zero joint velocity vector. Then, using the state supremum and infimum in section 6 , we illustrate the manipulator state performance. Finally, the local supremum and infimum in section 7 is calculated.

To provide an experimental test-bed, we have built a two degree-of-freedom planar revolute-jointed manipulator, shown schematically in Figure 1. The design variables of the manipulator consist of

The actuator torque set is

$$
\begin{aligned}
& l_{1}=0.303 \mathrm{~m} \\
& \mathrm{a}_{1}=0.196 \mathrm{~m} \\
& \mathrm{~m}_{1}=2.26 \mathrm{~kg} \\
& \mathrm{I}_{1}=0.129 \mathrm{~kg} \mathrm{~m}^{2}
\end{aligned}
$$

$$
\begin{aligned}
& l_{2}=0.254 \mathrm{~m} \\
& \mathrm{a}_{2}=0.0941 \mathrm{~m} \\
& \mathrm{~m}_{2}=0.177 \mathrm{~kg} \\
& \mathrm{I}_{2}=2.77 \times 10^{-3} \mathrm{~kg} \mathrm{~m}^{2}
\end{aligned}
$$

$$
\mathrm{T}=\left\{\tau|| \tau_{i} \mid \leq 30 . \mathrm{Nm}, i=1,2\right\},
$$

the joint-velocity set is

$$
\mathrm{v}=\left\{\dot{\mathrm{q}}|| \dot{q}_{i} \mid \leq 1.0 \mathrm{rad} / \mathrm{sec}, i=1,2\right\},
$$

and the workspace is

$$
\mathrm{W}=\left\{\mathbf{q} \mid 1 . \leq q_{2} \leq \pi \mathrm{rad}\right\} .
$$

We choose the manipulator position as $q=[0, \pi / 2]^{T}$. Our first step is to calculate the elements of matrices A, B for the manipulator position as follows;

$$
\begin{array}{llll}
a_{11}=0.000 & a_{12}=-58.666 & a_{21}=1.308 & a_{22}=-1.308 \\
b_{11}=-0.007 & b_{12}=-0.000 & b_{21}=-0.247 & b_{22}=-0.247
\end{array}
$$

Using equation (157), the state supremum at point $O^{\prime}$ of section 6 are calculated as $1761.73 \mathrm{~m} / \mathrm{sec}^{2}$. Since the parallelogram of section 4 is the state acceleration at point $O^{\prime}$, the supremum of the contribution, $\alpha_{\tau}$, of actuator torques in section 4 is also $1761.73 \mathrm{~m} / \mathrm{sec}^{2}$.

To obtain the state infimum, two conditions (158) and (159) are tested. Two conditions for our manipulator are both positive, and equation $(165)$ for case 4 is used to calculate the state infimum for $[0,1.57,0,0]^{T}$. The infimum for $[0,1.57,0,0]^{T}$ is $39.22 \mathrm{~m} / \mathrm{sec}^{2}$.

The local supremum is the supremum of the state acceleration located at point $O^{\prime}$ in Figure 17. From equation (166), the supremum of position $[0,1.57]^{T}$ is obtained as $1761.78 \mathrm{~m} / \mathrm{sec}^{2}$. To calculate the local infimum, two sets of state accelerations should be considered as in section 7 . The infimums for the state acceleration sets at point $O^{\prime}$, $F^{\prime}, E^{\prime}, G^{\prime}, I^{\prime}$, of section 6 are as follows:

$$
\begin{array}{lllll}
\text { point } \mathrm{O} & \text { point } \mathrm{F} & \text { point } \mathrm{E} & \text { point } \mathrm{G} & \text { point I } \\
1761.73 \mathrm{~m} / \mathrm{sec}^{2} & 1761.73 \mathrm{~m} / \mathrm{sec}^{2} & 1761.78 \mathrm{~m} / \mathrm{sec}^{2} & 1761.74 \mathrm{~m} / \mathrm{sec}^{2} & 1761.74 \mathrm{~m} / \mathrm{sec}^{2}
\end{array}
$$

Among these infimums, the minimum is the infimum as in equation (170). So, the local infimum for $[0,1.57]^{T}$ is $38.23 \mathrm{~m} / \mathrm{sec}^{2}$. 


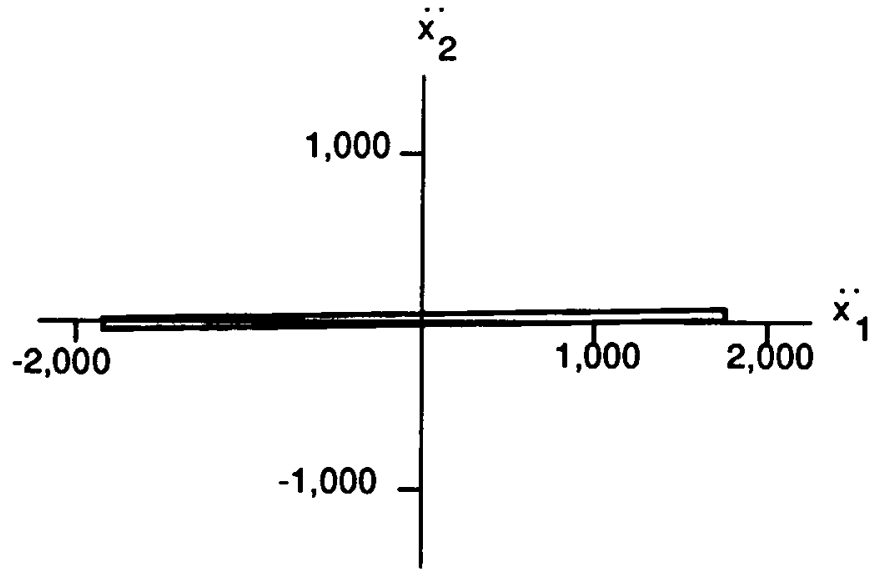

Figure 17: The acceleration set for a dynamic state $O$

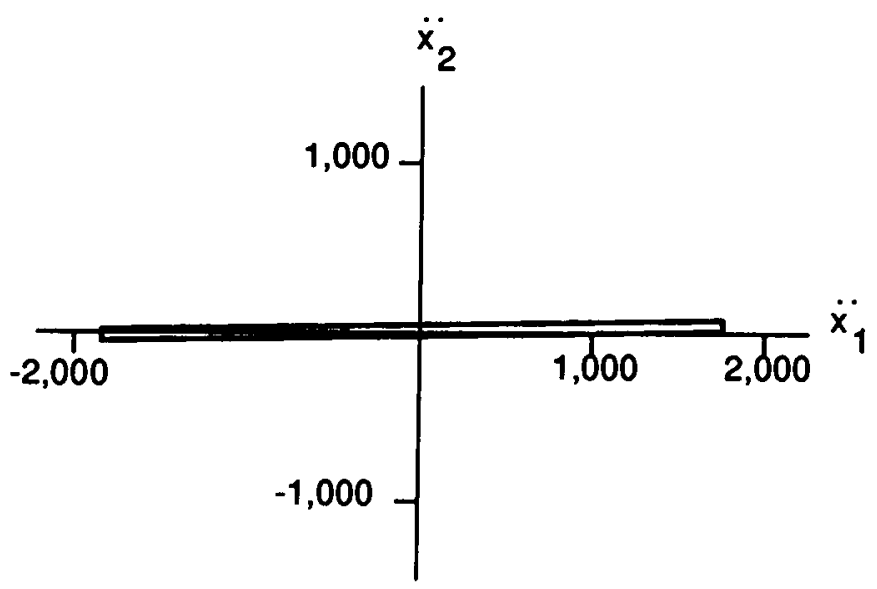

Figure 18: The local acceleration set 


\section{Equations of motion for a two-degree-of-freedom planar manipulator 1. Jacobian matrix}

The Jacobian matrix $\mathbf{J}$ has the following components:

$$
\begin{aligned}
& \mathrm{j}_{11}=-l_{1} \sin \mathrm{q}_{1}-l_{2} \sin \left(\mathrm{q}_{1}+\mathrm{q}_{2}\right) \\
& \mathrm{j}_{12}=-l_{2} \sin \left(\mathrm{q}_{1}+\mathrm{q}_{2}\right) \\
& \mathrm{j}_{21}=l_{1} \cos \mathrm{q}_{1}+l_{2} \cos \left(\mathrm{q}_{1}+\mathrm{q}_{2}\right) \\
& \mathrm{j}_{22}=l_{2} \cos \left(\mathrm{q}_{1}+\mathrm{q}_{2}\right)
\end{aligned}
$$

When this relationship is differentiated with respect to the time, we obtain the following equation.

$$
\ddot{\mathbf{x}}=\mathbf{J} \ddot{q}+\mathrm{J} \dot{\mathrm{q}}=\mathbf{J} \ddot{q}-\mathbf{E}(\dot{q})^{2}
$$

where $E$ is a (2×2) matrix which has the following elements:

$$
\begin{aligned}
& \mathrm{e}_{11}=l_{1} \cos \mathrm{q}_{1}+l_{2} \cos \left(\mathrm{q}_{1}+\mathrm{q}_{2}\right) \\
& \mathrm{e}_{12}=l_{2} \cos \left(\mathrm{q}_{1}+\mathrm{q}_{2}\right) \\
& \mathrm{e}_{21}=l_{1} \sin \mathrm{q}_{1}+l_{2} \sin \left(\mathrm{q}_{1}+\mathrm{q}_{2}\right) \\
& \mathrm{e}_{22}=l_{2} \sin \left(\mathrm{q}_{1}+\mathrm{q}_{2}\right)
\end{aligned}
$$

\section{Dynamic equation}

The dynamics of a two-degree-of-freedom planar manipulator is described by the following equation:

$$
\mathbf{D} \ddot{q}+V(\dot{q}]^{2}+p=\tau
$$

$D$ is a $(2 \times 2)$ matrix and the components are as follows:

$$
\begin{aligned}
& d_{11}=I_{1}+m_{1} a_{1}{ }^{2}+I_{2}+m_{2}\left(a_{2}{ }^{2}+2 a_{2} l_{1} \cos q_{2}+l_{1}{ }^{2}\right) \\
& d_{12}=I_{2}+m_{2}\left(a_{2}{ }^{2}+a_{2} l_{1} \cos q_{2}\right) \\
& d_{12}=d_{21} \\
& d_{22}=I_{2}+m_{2} a_{2}{ }^{2} .
\end{aligned}
$$

$\mathrm{V}$ is also a $(2 \times 2)$ matrix and has a following components:

$$
\begin{aligned}
& v_{11}=0 \\
& v_{12}=-v \\
& v_{21}=v \\
& v_{22}=0 .
\end{aligned}
$$

where

$$
\mathrm{v}=\mathrm{m}_{2} \mathrm{a}_{2} l_{1} \sin \mathrm{q}_{2} \text {. }
$$

$\mathrm{p}=\left[\mathrm{p}_{1} \mathrm{p}_{2}\right]^{T}$ is a vector with the rank 2 .

$$
\begin{aligned}
& p_{1}=m_{1} g a_{1} \sin q_{1}+m_{2} g\left[l_{1} \sin q_{1}+a_{2} \sin \left(q_{1}+q_{2}\right)\right] \\
& p_{2}=m_{2} g a_{2} \sin \left(q_{1}+q_{2}\right)
\end{aligned}
$$

where $\mathrm{g}$ is a gravitational constant.

\section{Acceleration equation}


The expression of the acceleration of the end-effector consists of three components as follows:

$$
\ddot{\mathbf{x}}=\mathbf{A} \tau+\mathbf{B}[\dot{\mathrm{q}}\}^{2}+\mathrm{C}
$$

where

$$
\begin{aligned}
& \mathbf{A}=\mathbf{J ~ D ~}^{-1} \\
& \mathbf{B}=-\mathbf{A} \mathbf{V}-\mathbf{E} \\
& \mathbf{C}=-\mathbf{A} \mathbf{p}
\end{aligned}
$$

$A$ is a (2×2) matrix and has the following components:

$$
\begin{aligned}
& a_{11}=\Delta\left(j_{11} d_{22}-j_{12} d_{12}\right) \\
& a_{12}=\Delta\left(-j_{11} d_{12}+j_{12} d_{11}\right) \\
& a_{21}=\Delta\left(j_{21} d_{22}-j_{22} d_{12}\right) \\
& a_{22}=\Delta\left(-j_{21} d_{12}+j_{22} d_{11}\right)
\end{aligned}
$$

where

$$
\Delta=\left[d_{11} d_{22}-d_{12}\right]^{-1}
$$

$B$ is also a (2×2) matrix and the elements are as follows:

$\mathrm{b}_{11}=-\mathrm{va}_{12}-\mathrm{e}_{11}$

$\mathrm{b}_{12}=\mathrm{va}_{11}-\mathrm{e}_{12}$

$b_{21}=-v a_{22}-e_{21}$

$\mathrm{b}_{22}=\mathrm{va}_{21}-\mathrm{e}_{22}$

$c=\left[c_{1} c_{2}\right]^{T}$ is a vector with the rank 2 .

$c_{1}=a_{11} p_{1}+a_{12} p_{2}$

$c_{2}=a_{21} p_{1}+a_{22} p_{2}$ 


\section{References}

1. Khatib,O. and Burdick,J., "Dynamic optimizationin manipulator design:The operational space formulation", , Proc.ASME Winter Annual Meeting, Miami, FL, Nov 1985.

2. Khatib,O. and Burdick,J., "Optimization of Dynamics in Manipulator Design: The Operationsl Space Formilation", Int J.Robot.Automation, Vol. 2, No. 2, Nov 1987, pp. 90-97.

3. Graettinger,T. and Krogh,B.H., "The Acceleration Radius: A Global Performance Measure for Robotic Manipulators", IEEE J.Robot.Automation, Vol. 4, No. 1, February 1988, pp. 60-69.

4. Kane,T. and Levinson D., Dynamics:Theory and Application, McGraw-Hill Inc., Vol. 2, 1985.

5. Yong-yil Kim, "Dynamics and Control of Multi-degree-of-freedom Mechanical Systems with Applications to Manipulator Design", Research Prospectus. 